\title{
The genus Alvania (Gastropoda: Rissoidae) along the Turkish Aegean coast with the description of a new species
}

\author{
Banu Bitlis ${ }^{1}$, Bilal Öztürk ${ }^{2}$ \\ ${ }^{1}$ Institute of Marine Sciences and Technology, Dokuz Eylul University, 35430 Inciralti, Izmir, Turkey. \\ (BB) (Corresponding author) E-mail: banu.bitlis@deu.edu.tr, banubitlis@yahoo.com. ORCID iD: \\ http://orcid.org/0000-0002-6542-8388 \\ ${ }^{2}$ Department Hydrobiology, Faculty of Fisheries, Ege University, 35100 Bornova, İzmir, Turkey. \\ (BO) E-mail: bilal.ozturk@ege.edu.tr. ORCID iD: http://orcid.org/0000-0002-5638-2496
}

\begin{abstract}
Summary: This study deals with the distribution of the species of the genus Alvania along the Turkish Aegean coast. The investigated material was collected from different habitats (soft and hard bottoms, and macrophyte beds) at a depth range of 0-875 m, at 39 stations along the Aegean coast of Turkey between 1995 and 2014. Among the analysed benthic material, 537 living specimens and 249 empty shells belonging to 20 species of the genus Alvania were obtained. Alvania marmarisensis is described as a new species. Alvania hispidula was recorded for the first time from the Turkish Aegean coast. Alvania mamillata was found to be the most widely distributed species in the study area, while Alvania colossophilus was the rarest one. Alvania cimicoides and Alvania testae were found in the deepest samples (between 93 and 875 m). Certain taxonomic and ecological characteristics of the identified species, along with photographs, are also provided.
\end{abstract}

Keywords: Aegean Sea; Turkey; Mollusca; Alvania; ecology; distribution.

El género Alvania (Gastropoda: Rissoidae) a lo largo de la costa turca del Egeo con la descripción de una nueva especie

Resumen: Este estudio trata de la distribución de las especies del género Alvania a lo largo de la costa turca del Egeo. El material investigado fue recolectado de diferentes hábitats (fondos blandos y duros y camas de macrófitas) a una profundidad de 0-875 m, en 39 estaciones a lo largo de la costa del Egeo turco entre 1995 y 2014. Entre el material bentónico analizado, 537 especímenes vivos y se obtuvieron 249 conchas vacías pertenecientes a 20 especies del género Alvania. Alvania marmarisensis se describe como nueva especie. Alvania hispidula se registró por primera vez desde la costa turca del Egeo. Se encontró que Alvania mamillata era la especie más ampliamente distribuida en el área de estudio, mientras que Alvania colossophilus era la más rara. Alvania cimicoides y Alvania testae se encontraron en las muestras más profundas (entre 93 y 875 m). También se proporcionan ciertas características taxonómicas y ecológicas de las especies identificadas, junto con fotografías.

Palabras clave: mar Egeo; Turquía; Mollusca; Alvania; ecología; distribución.

Citation/Como citar este artículo: Bitlis B., Öztürk B. 2017. The genus Alvania (Gastropoda: Rissoidae) along the Turkish Aegean coast with the description of a new species. Sci. Mar. 81(3): 395-411. doi: http://dx.doi.org/10.3989/scimar.04566.14A

LSID: urn:1sid:zoobank.org:pub:8080E3FC-7C9F-492C-97B0-BABCC05F92C6

Editor: J. Templado.

Received: October 10, 2016. Accepted: April 20, 2017. Published: July 3, 2017.

Copyright: (C) 2017 CSIC. This is an open-access article distributed under the terms of the Creative Commons Attribution (CC-by) Spain 3.0 License.

\section{INTRODUCTION}

The Aegean Sea is an ecologically distinct part of the Mediterranean Sea due to its peculiar hydrographic characteristics. It is an area where the brackish waters of the Black Sea $(17 \%$ ) merge with the saline waters of the eastern Mediterranean Sea (39\%o) (Öztürk et al. 2006). According to Kocataş and Bilecik (1992), ecological features such as temperature, salinity and nutrients fluctuate significantly in the southern Aegean Sea due to the influence of the eastern Mediterranean Sea, and in the northern Aegean Sea due to the influence of the Black Sea. This ecological variation affects the biota in the area. 
The genus Alvania Risso, 1826 belongs to the family Rissoidae, which is represented by a high number of genera and species in the northeast Atlantic and Mediterranean Sea (CLEMAM 2016). Alvania species are characterized by their typical clathrate sculpture on the teleoconch; ovate-conical shell with axial ribs, and spirals and nodules on their intersections varying according to the species. Alvania are differentiated from other rissoids by having either axial and spiral lines, or cords, on the surface of the teleoconch. The representatives of the genus have a worldwide distribution, with the exception of the Antarctic Ocean and sub-Antarctic regions (Ponder 1985), and most of the species (about $70 \%$ ) inhabit the littoral depths, although some of them can be found in deeper areas (Avila et al. 2012). The Alvania species inhabiting shallow depths feed mostly on periphyton of macrophytes, whereas some bathyal species [e.g. Alvania testae (Aradas and Maggiore, 1844), Alvania jeffreysi (Waller, 1864) and Alvania cimicoides (Forbes, 1844)] feed on the detritus and foraminifers (Fretter and Graham 1978, Ponder 1985).

Comprehensive revisions of the Rissooidea based on morphological characters have been published by Coan (1964) and Ponder (1985). Recently, a phylogenetic analysis of this family testing the diagnostic utility of morphological traits was conducted by Criscione et al. (2016).

The genus Alvania has been the subject of several studies in the Mediterranean Sea (e.g. van Aartsen 1982a, b, c, Amati 2014), where 74 Alvania species are present according to Avila et al. (2012). However, few works include taxonomic or ecological information on the representatives of this genus in the eastern Mediterranean (van Aartsen et al. 1989, Cecalupo and Quadri 1996).

Regarding the Turkish coasts, some information on the genus can be found in Forbes (1844), Oberling (1969-1971) and Buzzurro and Greppi (1996), while some species (A. amatii was reported by Oliverio (1986), A. datchaensis was reported by Amati and Oliverio (1987) and A. bozcaadensis and A. campanii were reported by Tisselli and Giunchi (2013).

The aim of this study is to contribute to the knowledge of the Alvania species distributed in the Turkish Aegean Sea, including information on their taxonomy, bathymetric distribution, preferred habitats and patterns of distribution. Special attention has been paid to the protoconch due to its importance as a taxonomic character. It can be used to deduce the type of larval development, which has implications for the potential dispersal capacity of larvae in the distribution areas. A multispiral protoconch (typically with more than 2 whorls) suggests planktotrophic larval development, while a paucispiral protoconch (fewer than 2 whorls) is indicative of non-planktotrophic larval development.

\section{MATERIALS AND METHODS}

The Alvania specimens investigated in this study were collected during various cruises or research projects conducted along the Aegean coast of Turkey between the years 1995 and 2014. The samples were taken from various substrates [sand, mud, mixture of

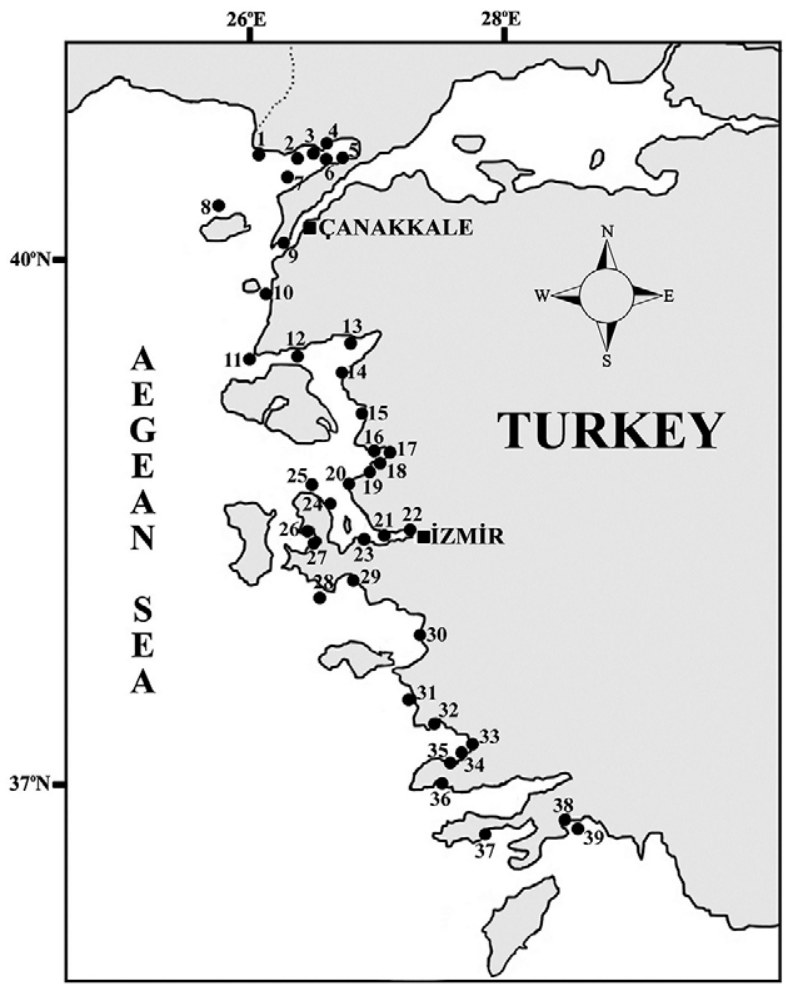

Fig. 1. - Map of the study area with the sampling stations.

sand and mud, coralligenous, algae (Padina pavonica, Cystoseira amentacea, C. crinita, C. foeniculacea f. schiffneri, C. spinosa, C. elegans, C. compressa, Halopteris scoparia, H. filicina, Ulva linza), phanerogams (Posidonia oceanica, Zostera noltei, Z. marina, Cymodocea nodosa), the sponge Aplysina aerophoba, the scleractinian coral Cladocora caespitosa, the fan shell Pinna nobilis and rocky bottoms] at depths ranging from 0 to $875 \mathrm{~m}$ at 39 stations (Fig. 1, Table 1). Benthic samples (10-875 m) were taken by means of a dredge and van Veen Grap, while the shallower water materials $(0-10 \mathrm{~m})$ were collected by snorkelling and hand picking. The sampled material was sieved through $0.5 \mathrm{~mm}$ mesh and the retained material was fixed in 5\% formalin solution. The Alvania specimens were separated from the other benthic materials using a stereomicroscope.

Some shell features of the investigated species, such as total height $(\mathrm{H})$ with standard errors, mean diameter (D) with standard errors, mean height (h) of the last whorl with standard errors and minimum and maximum values for each species [..... are presented in the following order: $\mathrm{H} \times \mathrm{D}-\mathrm{h} \mathrm{mm}[\ldots . . \mathrm{mm} ; . . . \mathrm{mm}]$.

The protoconch whorls of the investigated species were counted according to Warén (1974) and Verduin (1984), while the relevant specimens have been deposited in the museum of the Faculty of Fisheries (ESFM), Ege University (Izmir-Turkey).

\section{RESULTS}

As a result of the study of the material collected along the Turkish Aegean coast, we identified 537 specimens and 249 shells belonging to 20 species of 
Table 1. - Coordinates, sampling dates, depth ranges, biotopes, methods and location of the sampled sites.

\begin{tabular}{|c|c|c|c|c|c|c|}
\hline Sta. & Coordinates & Date & $\begin{array}{c}\text { Depth range } \\
(\mathrm{m})\end{array}$ & Biotope & $\begin{array}{l}\text { Sampling } \\
\text { method }\end{array}$ & Location \\
\hline 1 & $40^{\circ} 34^{\prime} 45^{\prime \prime} \mathrm{N}-26^{\circ} 09^{\prime} 25^{\prime \prime} \mathrm{E}$ & 04.08 .2000 & $5-8$ & Posidonia oceanica + Sand & Dredge & Saros Bay \\
\hline 2 & $40^{\circ} 32^{\prime} 45^{\prime \prime} \mathrm{N}-26^{\circ} 25^{\prime} 15^{\prime \prime} \mathrm{E}$ & 04.08 .2000 & 93 & Mud & & \\
\hline 3 & $40^{\circ} 33^{\prime} 00^{\prime \prime} \mathrm{N}-26^{\circ} 30^{\prime} 20^{\prime \prime} \mathrm{E}$ & 04.08 .2000 & 82 & Sandy mud & & \\
\hline 4.1 & $40^{\circ} 37^{\prime} 08^{\prime \prime} \mathrm{N}-26^{\circ} 38^{\prime} 17^{\prime \prime} \mathrm{E}$ & 03.08 .2000 & 32 & Muddy sand & & \\
\hline 4.2 & & 03.08 .2000 & 22 & Muddy sand & & \\
\hline 5.1 & $40^{\circ} 33^{\prime} 22^{\prime \prime} \mathrm{N}-26^{\circ} 44^{\prime} 38^{\prime \prime} \mathrm{E}$ & 05.07 .1995 & $0-0.5$ & Rock & By hand & \\
\hline 5.2 & & 05.07 .1995 & 1 & Padina pavonica & & \\
\hline 5.3 & & 05.07 .1995 & 2 & Cystoseira amentacea & & \\
\hline 6 & $40^{\circ} 31^{\prime} 07^{\prime \prime} \mathrm{N}-26^{\circ} 36^{\prime} 36^{\prime \prime} \mathrm{E}$ & 03.08 .2000 & 115 & Mud & Dredge & \\
\hline 7 & $40^{\circ} 26^{\prime} 36^{\prime \prime} \mathrm{N}-26^{\circ} 17^{\prime} 47^{\prime \prime} \mathrm{E}$ & 14.05 .2001 & 680 & Mud & & \\
\hline 8 & $40^{\circ} 17^{\prime} 06^{\prime \prime} \mathrm{N}-25^{\circ} 45^{\prime} 05^{\prime \prime} \mathrm{E}$ & 15.05 .2001 & 875 & Mud & & \\
\hline 9.1 & $40^{\circ} 03^{\prime} 07^{\prime \prime} \mathrm{N}-26^{\circ} 12^{\prime} 41^{\prime \prime} \mathrm{E}$ & 05.07 .1995 & 0.5 & Cystoseira spinosa & By hand & Çanakkale \\
\hline 9.2 & & 05.07 .1995 & 1 & Cystoseira foeniculace a f. schiffneri & & \\
\hline 9.3 & & 05.07 .1995 & 0.5 & Padina pavonica & & \\
\hline 9.4 & & 05.07 .1995 & $0.8-1.5$ & Zostera noltei & & \\
\hline 9.5 & & 05.07 .1995 & $0-1$ & Rock & & \\
\hline 10 & $39^{\circ} 46^{\prime} 57^{\prime \prime} \mathrm{N}-26^{\circ} 09^{\prime} 24^{\prime \prime} \mathrm{E}$ & 26.09 .1997 & $0.5-1$ & Zostera noltei & & Ezine \\
\hline 11 & $39^{\circ} 28^{\prime} 25^{\prime \prime} \mathrm{N}-26^{\circ} 03^{\prime} 17^{\prime \prime} \mathrm{E}$ & 25.08 .2011 & 23 & Posidonia oceanica & Grap & Bababurnu \\
\hline 12 & $39^{\circ} 29^{\prime} 06^{\prime \prime} \mathrm{N}-26^{\circ} 20^{\prime} 19^{\prime \prime} \mathrm{E}$ & 16.07 .1998 & 10 & Cladocora caespitosa & By hand & Behramkale \\
\hline 13.1 & $39^{\circ} 33^{\prime} 37^{\prime \prime} \mathrm{N}-26^{\circ} 49^{\prime} 52^{\prime \prime} \mathrm{E}$ & 06.07 .1995 & 0.6 & Padina pavonica & & Altınoluk \\
\hline 13.2 & & 06.07 .1995 & $0-1$ & Rock & & \\
\hline 14.1 & $39^{\circ} 21^{\prime} 30^{\prime \prime} \mathrm{N}-26^{\circ} 38^{\prime} 02^{\prime \prime} \mathrm{E}$ & 06.07 .1995 & $0-0.5$ & Rock & & Cunda Island \\
\hline 14.2 & & 06.07 .1995 & 0.4 & Padina pavonica & & (Ayvalık) \\
\hline 14.3 & & 06.07 .1995 & 2 & Zostera noltei & & \\
\hline 14.4 & & 06.07 .1995 & 0.5 & Cystoseira elegans & & \\
\hline 15.1 & $39^{\circ} 07^{\prime} 35^{\prime \prime} \mathrm{N}-26^{\circ} 50^{\prime} 05^{\prime \prime} \mathrm{E}$ & 07.07 .1995 & $0-1$ & Rock & & Dikili \\
\hline 15.2 & & 07.07 .1995 & 1 & Padina pavonica & & \\
\hline 15.3 & & 07.07 .1995 & 1 & Cystoseira crinita & & \\
\hline 15.4 & & 17.03 .2005 & 10 & Sand & Grap & \\
\hline 16.1 & $38^{\circ} 51^{\prime} 04^{\prime \prime} \mathrm{N}-26^{\circ} 54^{\prime} 56^{\prime \prime} \mathrm{E}$ & 07.03 .2003 & 50 & Coralligenous & Dredge & Çandarlı Bay \\
\hline 16.2 & $38^{\circ} 55^{\prime} 29^{\prime \prime} \mathrm{N}-26^{\circ} 49^{\prime} 30^{\prime \prime} \mathrm{E}$ & 07.03 .2003 & 46 & Coralligenous sand & & \\
\hline 16.3 & $38^{\circ} 55^{\prime} 36^{\prime \prime} \mathrm{N}-26^{\circ} 49^{\prime} 10^{\prime \prime} \mathrm{E}$ & 22.11.2002 & 50 & Mud & & \\
\hline 16.4 & $38^{\circ} 55^{\prime} 36^{\prime \prime} \mathrm{N}-26^{\circ} 49^{\prime} 10^{\prime \prime} \mathrm{E}$ & 07.03 .2003 & $56-62$ & Coralligenous sand & & \\
\hline 17.1 & $38^{\circ} 53^{\prime} 13^{\prime \prime} \mathrm{N}-27^{\circ} 03^{\prime} 52^{\prime \prime} \mathrm{E}$ & 07.07 .1995 & $3-4$ & Pinna nobilis & By hand & Şakran \\
\hline 17.2 & & 07.07 .1995 & 2.5 & Zostera marina & & \\
\hline 18 & $38^{\circ} 48^{\prime} 49^{\prime \prime} \mathrm{N}-26^{\circ} 57^{\prime} 04^{\prime \prime} \mathrm{E}$ & 28.08 .2011 & 13 & Posidonia oceanica & Grap & Aliağa \\
\hline 19 & $38^{\circ} 46^{\prime} 12^{\prime \prime} \mathrm{N}-26^{\circ} 55^{\prime} 27^{\prime \prime} \mathrm{E}$ & 04.10 .2001 & 12 & Posidonia oceanica & Dredge & Nemrut Bay \\
\hline 20.1 & $38^{\circ} 40^{\prime} 45^{\prime \prime} \mathrm{N}-26^{\circ} 44^{\prime} 26^{\prime \prime} \mathrm{E}$ & 07.07.1995-08.05.1996 & 1 & Halopteris scoparia & By hand & Foça \\
\hline 20.2 & & 08.05 .1996 & $0-0.5$ & Rock & & \\
\hline 20.3 & & 07.07 .1995 & 0.5 & Padina pavonica & & \\
\hline 21.1 & $38^{\circ} 25^{\prime} 41^{\prime \prime} \mathrm{N}-27^{\circ} 03^{\prime} 23^{\prime \prime} \mathrm{E}$ & 28.04 .2013 & 23 & Mud & Grap & İzmir Bay \\
\hline 21.2 & $38^{\circ} 25^{\prime} 27^{\prime \prime} \mathrm{N}-26^{\circ} 58^{\prime} 46^{\prime \prime} \mathrm{E}$ & 08.10 .2012 & 15 & Mud & & \\
\hline 22 & $38^{\circ} 29^{\prime} 16^{\prime \prime} \mathrm{N}-26^{\circ} 39^{\prime} 26^{\prime \prime} \mathrm{E}$ & $13.02 .2012-13.02 .2002$ & 12 & Posidonia oceanica & & \\
\hline 23.1 & $38^{\circ} 22^{\prime} 15^{\prime \prime} \mathrm{N}-26^{\circ} 46^{\prime} 59^{\prime \prime} \mathrm{E}$ & 14.06 .1995 & $0.5-1$ & Padina pavonica & By hand & Urla \\
\hline 23.2 & & 14.06 .1995 & 0.5 & Ulva linza & & \\
\hline 23.3 & & 14.06 .1995 & 0.5 & Zostera marina & & \\
\hline 23.4 & & 14.06 .1995 & 0.7 & Cystoseira crinita & & \\
\hline 24.1 & $38^{\circ} 39^{\prime} 49^{\prime \prime} \mathrm{N}-26^{\circ} 29^{\prime} 22^{\prime \prime} \mathrm{E}$ & 20.09.1995-09.05.1996 & $0-2.5$ & Rock & & Karaburun \\
\hline 24.2 & & 20.09 .1995 & 1.5 & Halopteris filicina & & \\
\hline 24.3 & & 20.09.1995-09.05.1996 & 4 & Posidonia oceanica & & \\
\hline 24.4 & & 09.05 .1996 & $0.5-1$ & Cystoseira crinita & & \\
\hline 25 & $38^{\circ} 40^{\prime} 45^{\prime \prime} \mathrm{N}-26^{\circ} 30^{\prime} 51^{\prime \prime} \mathrm{E}$ & 01.04 .2010 & 74 & Muddy sand & Grap & \\
\hline 26.1 & $38^{\circ} 27^{\prime} 24^{\prime \prime} \mathrm{N}-26^{\circ} 27^{\prime} 18^{\prime \prime} \mathrm{E}$ & 09.05.2007-20.02.2013 & $50-62$ & Muddy sand & & Ildır Bay \\
\hline 26.2 & $38^{\circ} 27^{\prime} 12^{\prime \prime} \mathrm{N}-26^{\circ} 27^{\prime} 18^{\prime \prime} \mathrm{E}$ & 08.01 .2009 & 52 & Sandy mud & & \\
\hline 26.3 & $38^{\circ} 27^{\prime} 12^{\prime \prime} \mathrm{N}-26^{\circ} 27^{\prime} 09^{\prime \prime} \mathrm{E}$ & 07.03.2007-26.03.2009 & $50-65$ & Sandy mud & & \\
\hline 26.4 & $38^{\circ} 27^{\prime} 24^{\prime \prime} \mathrm{N}-26^{\circ} 27^{\prime} 09^{\prime \prime} \mathrm{E}$ & 21.09.2006-16.09.2009 & $49-61$ & Sandy mud & & \\
\hline 27.1 & $38^{\circ} 23^{\prime} 27^{\prime \prime} \mathrm{N}-26^{\circ} 26^{\prime} 54^{\prime \prime} \mathrm{E}$ & $09.06 .2003-03.10 .2007$ & $18-25$ & P. oceanica + Sand & Dredge & \\
\hline 27.2 & & $22.02 .2001-03.10 .2007$ & $45-60$ & Sand & & \\
\hline 27.3 & & $03.10 .2007-25.06 .2009$ & $25-46$ & Sandy mud & & \\
\hline 27.4 & & 07.03.2007-31.10.2012 & $30-50$ & P. oceanica+Mud & & \\
\hline 27.5 & & $03.10 .2007-20.02 .2013$ & $10-50$ & P. oceanica+Muddy sand & & \\
\hline 27.6 & & $07.05 .2004 / 27.05 .2004$ & 5 & Zostera marina+Sand & & \\
\hline 28 & $38^{\circ} 06^{\prime} 15^{\prime \prime} \mathrm{N}-26^{\circ} 27^{\prime} 22^{\prime \prime} \mathrm{E}$ & 14.09 .2000 & 195 & Mud & & Çeşme \\
\hline 29.1 & $38^{\circ} 11^{\prime} 51^{\prime \prime} \mathrm{N}-26^{\circ} 46^{\prime} 45^{\prime \prime} \mathrm{E}$ & 22.06 .1995 & 0.3 & Padina pavonica & By hand & Siğacik \\
\hline 29.2 & & 22.06 .1995 & $0-1$ & Rock & & \\
\hline 29.3 & & 22.06 .1995 & 0.5 & Cystoseira compressa & & \\
\hline 29.4 & & 22.06 .1995 & $0.5-2$ & Zostera marina & & \\
\hline 30.1 & $37^{\circ} 51^{\prime} 34^{\prime \prime} \mathrm{N}-27^{\circ} 14^{\prime} 49^{\prime \prime} \mathrm{E}$ & 14.06 .1996 & 0.5 & Padina pavonica & & Kuşadası \\
\hline 30.2 & & $23.07 .1995-14.06 .1996$ & 1 & Halopteris filicina & & \\
\hline 31 & $37^{\circ} 25^{\prime} 17^{\prime \prime} \mathrm{N}-27^{\circ} 12^{\prime} 21^{\prime \prime} \mathrm{E}$ & 01.09 .2011 & 36 & Muddy sand & Grap & Didim \\
\hline 32.1 & $37^{\circ} 21^{\prime} 55^{\prime \prime} \mathrm{N}-27^{\circ} 21^{\prime} 37^{\prime \prime} \mathrm{E}$ & 23.07.1995 & 1 & Cystoseira crinita & By hand & \\
\hline 32.2 & & 23.07 .1995 & $0-1$ & Rock & & \\
\hline 32.3 & & 23.07 .1995 & $3-4$ & Pinna nobilis & & \\
\hline 32.4 & & 23.07 .1995 & $1-2$ & Aplysina aerophoba & & \\
\hline 32.5 & $37^{\circ} 22^{\prime} 02^{\prime \prime} \mathrm{N}-27^{\circ} 22^{\prime} 11^{\prime \prime} \mathrm{E}$ & 01.09 .2011 & 24 & Sand & Grap & \\
\hline
\end{tabular}


Table 1 (Cont.). - Coordinates, sampling dates, depth ranges, biotopes, methods and location of the sampled sites.

\begin{tabular}{|c|c|c|c|c|c|c|}
\hline Sta. & Coordinates & Date & $\begin{array}{l}\text { Depth range } \\
(\mathrm{m})\end{array}$ & Biotope & $\begin{array}{l}\text { Sampling } \\
\text { method }\end{array}$ & Location \\
\hline 33.1 & $37^{\circ} 13^{\prime} 32^{\prime \prime} \mathrm{N}-27^{\circ} 35^{\prime} 20^{\prime \prime} \mathrm{E}$ & 22.07.1995 & $0-1$ & Rock & By hand & Güllük \\
\hline 33.2 & & 22.07 .1995 & 2 & Cymodocea nodosa & & \\
\hline 33.3 & & 22.07 .1995 & 1 & Cystoseira foeniculacea $\mathrm{f}$. schiffneri & & \\
\hline 34 & $37^{\circ} 08^{\prime} 33^{\prime \prime} \mathrm{N}-27^{\circ} 30^{\prime} 12^{\prime \prime} \mathrm{E}$ & $08.05 .2002-21.10 .2001$ & $9.5-13.5$ & P. oceanica+Sand & Dredge & Salih Island \\
\hline 35.1 & $37^{\circ} 05^{\prime} 04^{\prime \prime} \mathrm{N}-27^{\circ} 27^{\prime} 59^{\prime \prime} \mathrm{E}$ & 22.07 .1995 & $0-0.5$ & Rock & By hand & Torba \\
\hline 35.2 & & 22.07 .1995 & 0.7 & Cystoseira crinita & & \\
\hline 36.1 & $37^{\circ} 01^{\prime} 16^{\prime \prime} \mathrm{N}-27^{\circ} 26^{\prime} 29^{\prime \prime} \mathrm{E}$ & 22.07 .1995 & $0-1$ & Rock & & Bodrum \\
\hline 36.2 & & 22.07 .1995 & 0.5 & Padina pavonica & & \\
\hline 36.3 & & 22.07 .1995 & 1.5 & Cystoseira crinita & & \\
\hline 36.4 & & 22.07 .1995 & 0.8 & Halopteris scoparia & & \\
\hline 36.5 & & 16.07.1998 & 10 & Posidonia oceanica & & \\
\hline 37.1 & $36^{\circ} 43^{\prime} 04^{\prime \prime} \mathrm{N}-27^{\circ} 41^{\prime} 33^{\prime \prime} \mathrm{E}$ & 20.07 .1995 & $0-1$ & Rock & & Datça \\
\hline 37.2 & & 20.07.1995 & 1.5 & Cystoseira crinita & & \\
\hline 37.3 & & 20.07 .1995 & 0.7 & Padina pavonica & & \\
\hline 38.1 & $36^{\circ} 46^{\prime} 09^{\prime \prime} \mathrm{N}-28^{\circ} 15^{\prime} 25^{\prime \prime} \mathrm{E}$ & 21.07 .1995 & 1 & Cystoseira amentacea & & Turunç \\
\hline 38.2 & & 21.07 .1995 & $0-3$ & Rock & & \\
\hline 39.1 & $36^{\circ} 45^{\prime} 19^{\prime \prime} \mathrm{N}-28^{\circ} 21^{\prime} 14^{\prime \prime} \mathrm{E}$ & 01.10 .2014 & 99 & Mud & Dredge & Marmaris \\
\hline 39.2 & $36^{\circ} 46^{\prime} 24^{\prime \prime} \mathrm{N}-28^{\circ} 20^{\prime} 42^{\prime \prime} \mathrm{E}$ & 01.10 .2014 & 80 & Mud & & \\
\hline
\end{tabular}

the genus Alvania. One of them is considered as a new species and is here described and figured for the first time. Table 2 provides a list of the identified Alvania species, the number of living specimens and empty shells obtained of each one, and the stations, habitats and deep range where they were found.

\section{Systematics}

Superorder CAENOGASTROPODA Cox, 1960 Superfamily RISSOOIDEA Gray, 1847 Family RISSOIDAE Gray, 1847 Genus Alvania Risso, 1826

Type species: Alvania europea Risso 1826: 142, pl. 9, Fig. $116=$ Alvania cimex (Linnaeus, 1758) (Turbo), by subsequent designation Nevill 1885: 105.

Alvania amatii Oliverio, 1986

(Fig. 2A, B)

Alvania amatii Oliverio, 1986

Mean dimensions ( 1 specimen and 3 shells): $2.02( \pm 0.22) \times 1.23$ $( \pm 0.08)-1.42( \pm 0.12) \mathrm{mm}[1.5 \times 1.0-1.1 \mathrm{~mm} ; 2.6 \times 1.4-1.7 \mathrm{~mm}]$.

Remarks. Alvania amatii is characterized by its smaller shell dimensions and paucispiral protoconch having 5 spiral threads.

Distribution. The species seems to be an eastern Mediterranean endemic (Oliverio 1986, van Aartsen et al. 1989). Turkish coasts: Aegean Sea (Oliverio 1986, van Aartsen and Kinzelbach 1990) and Levantine Sea (Buzzurro and Greppi 1996, Bitlis Bakir et al. 2012).

\section{Alvania aspera (Philippi, 1844)}

(Fig. 2C, D)

Rissoa aspera Philippi, 1844

Mean dimensions $(9$ specimens and 1 shell): $1.54( \pm 0.32) \times 1.14$ $( \pm 0.15)-1.11( \pm 0.20) \mathrm{mm}[0.7 \times 0.6-0.6 \mathrm{~mm} ; 3.7 \times 2.1-2.4 \mathrm{~mm}]$.

Remarks. The medium-sized and strong shell, thick axial ribs wider than interspaces on the last whorl and paucispiral protoconch are some of the evident diagnostic features of $A$. aspera.

Distribution. A. aspera is an eastern Mediterranean endemic (Bogi et al. 1989, Barash and Danin 1992, Zenetos and van Aartsen 1995). Turkish coasts: Levantine and Aegean coasts (Demir 2003, Öztürk et al. 2008).

Alvania beanii (Hanley in Thorpe, 1844)

(Figs 2E, F, 3D, E, 7K)

Cingula beanie Hanley in Thorpe, 1844

Mean dimensions ( 39 specimens and 11 shells): $2.83( \pm 0.12) \times 1.62$ $( \pm 0.05)-1.86( \pm 0.07) \mathrm{mm}[2.8 \times 1.6-1.9 \mathrm{~mm} ; 3.9 \times 2.1-2.4 \mathrm{~mm}]$.

Remarks. A. beanii has a multispiral protoconch. It differs from the similar A. hispidula in its greater number of spiral cords on the penultimate and last whorls of the teleoconch.

Distribution. Arctic Ocean (Hudenbick and Warén 1969), northeast Atlantic Ocean and Mediterranean Sea (Oliverio et al. 1986, Scaperrotta et al. 2011). Turkish coasts: Aegean Sea (Demir 2003), Sea of Marmara (Ostroumoff 1894, 1896) and Levantine Sea (Bitlis Bakır and Öztürk 2016).

Alvania bozcaadensis Tisselli and Giunchi, 2013 (Fig. 2. G, H)

Alvania bozcaadensis Tisselli and Giunchi, 2013

Dimensions of two specimens: $1.8 \times 1.2-1.3,1.0 \times 0.8-0.9 \mathrm{~mm}$.

Remarks. The species can be confused with $A$. lanciae (Calcara, 1845) and with the juveniles of $A$. discors, but $A$. bozcaadensis has no microsculpture on the surface of its protoconch and teleoconch, whereas A. lanciae bears microsculpture on both its protoconch and teleoconch. It differs from $A$. discors because the protoconch of the later species has a multispiral proto- 
Table 2. - List of Alvania species collected during this study, stations, number of living specimens and empty shells, depth range and biotopes (S, sand; M, mud; Ms, muddy sand; Sm, sandy mud; Cor, coralligenous; Po, Posidonia oceanica; Zm, Zostera marina; Zn, Zostera noltei; Cn, Cymodocea nodosa; Pp, Padina pavonica; Ca, Cystoseira amentacea; Ccr, C. crinita; Cf, C. foeniculacea f. schiffneri; Cs, C. spinosa; Ce, C. elegans; Cco, C. compressa; Hs, Halopteris scoparia; Hf, H. filicina; Ul, Ulva linza; Aa, Aplysina aerophoba; Clc, Cladocora caespitosa; Pn, Pinna nobilis; R, rocky).

\begin{tabular}{|c|c|c|c|c|}
\hline Species & $\begin{array}{l}\text { Number of living } \\
\text { specimens and empty } \\
\text { shells }\end{array}$ & Biotope & $\begin{array}{l}\text { Depth range } \\
(\mathrm{m})\end{array}$ & Stations \\
\hline Alvania amatii & $1 \mathrm{spc} ., 3 \mathrm{sh}$. & R, Hf & $0-1$ & Sta. $30.2,1$ sh.; sta. $33.1,1$ sp. +1 sh.; sta. $36.1,1$ sh. \\
\hline Alvania aspera & 9 spc., 1 sh. & $\mathrm{Ccr}, \mathrm{Ce}, \mathrm{Pp}$ & $0.5-1.5$ & Sta. $14.4,1$ sp.; sta. $23.1,1$ sp.; sta. $37.2,7$ sp. +1 sh. \\
\hline Alvania beanii & 39 spc., 11 sh. & Po, Cor, S, M, Ms & $23-100$ & $\begin{array}{l}\text { Sta. } 11,18 \text { sp. }+7 \text { sh.; sta. } 16.1,2 \text { sp.; sta. } 16.2,1 \text { sp.; } \\
\text { sta. } 16.4,1 \text { sp.; sta. } 25,8 \text { sp.; sta. } 31 \text {, } 1 \text { sh.; sta. } 39.1,8 \\
\text { sp.+3 sh.; sta. } 39.2,1 \text { sp. }\end{array}$ \\
\hline Alvania bozcaadensis & $2 \mathrm{spc}$. & Po, Aa & $1-4$ & Sta. $24.3,1$ sp.; sta. $32.4,1$ sp. \\
\hline Alvania cancellata & 26 spc., 5 sh. & $\begin{array}{l}\text { Po, S, Ca, Clc, Cor, } \\
\text { M, Ms }\end{array}$ & $2-74$ & $\begin{array}{l}\text { Sta. } 1,2 \text { sp.; sta. } 5.3,1 \text { sp.+2 sh.; sta. } 12,4 \text { sp.+3 sh.; } \\
\text { sta. } 15.4,2 \text { sp.; sta. } 16.1,3 \text { sp.; sta. } 16.4,6 \text { sp.; sta. } 19 \text {, } \\
2 \text { sp.; sta. } 25,1 \text { sp.; sta. } 27.1,2 \text { sp.; sta. } 34,3 \text { sp. }\end{array}$ \\
\hline Alvania carinata & $2 \mathrm{spc}$ & Po, $\mathrm{S}$ & $5-8$ & Sta. $1,2 \mathrm{sp}$ \\
\hline Alvania cimex & 31 spc., 35 sh. & $\begin{array}{l}\mathrm{Ca}, \mathrm{Clc}, \mathrm{R}, \mathrm{Zn}, \mathrm{S}, \mathrm{Hf} \\
\mathrm{Hs}, \mathrm{Pp}, \mathrm{Po}, \mathrm{Ccr}, \mathrm{Cco} \\
\text { Aa, Pn, M, Ms }\end{array}$ & $0-30$ & $\begin{array}{l}\text { Sta. 5.3, } 1 \text { sp.; sta. } 12,5 \text { sp.; sta. } 14.1,3 \text { sp.; sta. } 14.3,2 \\
\text { sh.; sta. } 15.1,1 \text { sp.+1 sh.; sta. } 15.4,3 \text { sp.; sta. } 20.1,1 \text { sp.; } \\
\text { sta. } 20.2 \text {, } 4 \text { sp.+3 sh.; sta. } 20.3,2 \text { sp.+5 sh.; sta. } 21.2,1 \\
\text { sp.; sta. } 22,3 \text { sp.; sta. } 23.1,2 \text { sp.+3 sh.; sta. } 23.4: 2 \text { sh.; } \\
\text { sta. } 24.4: 1 \text { sh.; sta. } 27.4,1 \text { sp.+1 sh.; sta. } 27.5,5 \text { sh.; } \\
\text { sta. 29.2, } 1 \text { sh.; sta. } 29.3 \text {, } 2 \text { sp.; sta. } 30.2 \text {, } 1 \text { sh.; sta. } 32.1 \text {, } \\
5 \text { sh.; sta. } 32.3,2 \text { sp.+2 sh.; sta. } 32.4,3 \text { sh. }\end{array}$ \\
\hline Alvania cimicoides & 3 spc., 11 sh. & M & $195-875$ & Sta. 7,1 sh.; sta. 8,6 sh.; sta. 28,3 sp. +4 sh. \\
\hline Alvania colossophilus & $1 \mathrm{sh}$. & Po, M & 30 & Sta. $27.4,1$ sh. \\
\hline Alvania datchaensis & 52 spc., 17 sh. & $\begin{array}{l}\text { R, Po, Ccr, Cn, Pp } \\
\text { Ca, S }\end{array}$ & $0-13.5$ & $\begin{array}{l}\text { Sta. } 15.1,3 \text { sp.; sta. } 24.3,5 \text { sp.; sta. } 32.1,3 \text { sp.; sta. } \\
\text { 32.2, } 2 \text { sp.; sta. } 33.1 \text {, } 4 \text { sp.; sta. } 33.2 \text {, } 2 \text { sh.; sta. } 34,1 \\
\text { sp.; sta. } 35.1,2 \text { sp.; sta. } 35.2,2 \text { sp.; sta. } 36.1,4 \text { sp.+1 } \\
\text { sh.; sta. } 36.2 \text {, } 3 \text { sp.; sta. } 36.3,12 \text { sp.; sta. } 37.1 ; 3 \text { sp.; sta. } \\
\text { 37.2, } 3 \text { sp.+13 sh.; sta. } 38.1,2 \text { sp.+1 sh.; sta. } 38.2 \text {, } 3 \text { sp. }\end{array}$ \\
\hline Alvania discors & 218 spc., $82 \mathrm{sh}$. & $\begin{array}{l}\text { R, Pp, Cs, Zn, Ccr, } \\
\text { Pn, Ul, Hf, Cf, Cco, } \\
\text { Cn, Cs, Ca, Po, Hs }\end{array}$ & $0-4$ & $\begin{array}{l}\text { Sta. } 5.1,20 \text { sp.; sta. } 5.2,2 \text { sp.; sta. } 5.3,12 \text { sh.; sta. } 9.1 \text {, } \\
19 \text { sp.; sta. } 9.2,3 \text { sp.+41 sh.; sta. } 9.3,4 \text { sh.; sta. } 9.4 \text {, } \\
3 \text { sh.; sta. } 9.5,1 \text { sp.; sta. } 13.2,2 \text { sh.; sta. } 14.1,4 \text { sp.; } \\
\text { sta. } 14.2,1 \text { sh.; sta. } 14.3 \text {, } 4 \text { sh.; sta. } 15.3,1 \text { sh.; sta. } \\
17.1,1 \text { sp.; sta. } 23.1,2 \text { sp.; sta. } 23.2,1 \text { sh.; sta. } 24.1 \text {, } \\
3 \text { sp.+1 sh.; sta. } 24.2,5 \text { sp.; sta. } 24.3,47 \text { sp.; sta. } 24.4 \text {, } \\
14 \text { sp.+5 sh.; sta. } 29.1,2 \text { sp.; sta. } 29.2,1 \text { sp.+3 sh.; sta. } \\
29.3,35 \text { sp.+1 sh.; sta. } 30.1,2 \text { sh.; sta. } 30.2,24 \text { sp.; } \\
\text { sta. } 32.2,8 \text { sp.; sta. } 33.1,2 \text { sp.; sta. } 33.2,2 \text { sp.; sta. } \\
33.3,1 \text { sh.; sta. } 35.1,3 \text { sp.; sta. } 35.2,14 \text { sp.; sta. } 36.4 \text {, } \\
1 \text { sp.; sta. } 37.1,2 \text { sp.; sta. } 37.2,3 \text { sp. }\end{array}$ \\
\hline Alvania geryonia & 26 spc., 24 sh. & $\begin{array}{l}\text { Ms, Ca, Po, R, S, } \\
\text { Cor, M, Sm, Pn, Zm }\end{array}$ & $3-58$ & $\begin{array}{l}\text { Sta. 4.2, } 1 \text { sp.; sta. } 5.3,1 \text { sp.; sta. } 11,1 \text { sp.+3 sh.; sta. } \\
14.1,1 \text { sp.; sta. } 16.2,3 \text { sp.; sta. } 18,7 \text { sh.; sta. } 21.1,1 \\
\text { sh.; sta. } 22,1 \text { sp.; sta. } 26.1,1 \text { sh.; sta. } 26.3,1 \text { sp.+3 sh.; } \\
\text { sta. } 26.4,2 \text { sh.; sta. } 27.1,3 \text { sp.+1 sh.; sta. } 27.2,3 \text { sp.+2 } \\
\text { sh.; sta. } 27.3,4 \text { sp.+3 sh.; sta. } 27.4,1 \text { sh.; sta. } 27.6,3 \\
\text { sp.; sta. } 32.3,2 \text { sp.; sta. } 34,2 \text { sp. }\end{array}$ \\
\hline Alvania hispidula & 2 spc., 4 sh. & Po, M, Ms & $23-100$ & Sta. 11,3 sh.; sta. 25,1 sp.; sta. $39.1,1$ sp.+1 sh. \\
\hline Alvania lactea & $11 \mathrm{spc} ., 6 \mathrm{sh}$. & $\mathrm{R}, \mathrm{Zn}, \mathrm{Clc}, \mathrm{S}, \mathrm{Hs}, \mathrm{Pp}$ & $0-10$ & $\begin{array}{l}\text { Sta. } 5.1,1 \text { sh.; sta. } 9.4,1 \text { sp.; sta. } 10,2 \text { sp.; sta. } 12,1 \\
\text { sh.; sta. } 14.3,1 \text { sh.; sta. } 15.1,1 \text { sp. }+1 \text { sh.; sta. } 15.4,4 \\
\text { sp.; sta. } 20.1,2 \text { sh.; sta. } 29.1,1 \text { sp.; sta. } 32.2,1 \text { sp.; sta. } \\
35.1,1 \text { sp. }\end{array}$ \\
\hline Alvania lineata & $4 \mathrm{spc}$ & $\mathrm{R}, \mathrm{Ca}, \mathrm{Po}$ & $0-4$ & Sta. 5.1, 1 sp.; sta. 5.3, 2 sp.; sta. 24.3, 1 sp. \\
\hline Alvania mamillata & 32 spc., 23 sh. & $\begin{array}{l}\mathrm{R}, \mathrm{Pp}, \mathrm{Ca}, \mathrm{Cf}, \mathrm{Zn} \\
\mathrm{Ce}, \mathrm{Zm}, \mathrm{S}, \mathrm{Hf}, \mathrm{Pn} \\
\text { Po, Ccr }\end{array}$ & $0-13$ & $\begin{array}{l}\text { Sta. } 5.1,1 \text { sp.+1 sh.; sta. } 5.2,2 \text { sp.; sta. } 5.3,7 \text { sp.; sta. } \\
\text { 9.2, } 3 \text { sh.; sta. } 9.4,2 \text { sh.; sta. } 10,2 \text { sh.; sta. } 14.1 \text {, } 1 \text { sh.; } \\
\text { sta. } 14.2 \text {, } 2 \text { sh.; sta. } 14.3 \text {, } 1 \text { sp.; sta. } 14.4,2 \text { sp.; sta. } \\
\text { 15.1, } 1 \text { sp.; sta. } 17.2,1 \text { sh.; sta. } 18,1 \text { sp.; sta. } 23.3,2 \\
\text { sp.; sta. } 24.1,1 \text { sh.; sta. } 27.6,1 \text { sp.; sta. } 29.4,1 \text { sh.; sta. } \\
\text { 30.2, } 11 \text { sp.+2 sh.; sta. } 32.2,2 \text { sh; sta. } 32.3,1 \text { sh.; sta. } \\
\text { 33.1, } 1 \text { sh.; sta. } 35.1,1 \text { sp.; sta. } 36.2,1 \text { sh.; sta. } 36.5,1 \\
\text { sh.; sta. } 37.2,1 \text { sh.; sta. } 37.3,1 \text { sp.; sta. } 38.2,1 \text { sp. }\end{array}$ \\
\hline Alvania punctura & 42 spc., 24 sh. & Sm, Ms, M, S, Po & $10-100$ & $\begin{array}{l}\text { Sta. } 3,8 \text { sp.; sta. } 4.1,5 \text { sp.; sta. } 16.3,2 \text { sp.; sta. } 25,20 \\
\text { sp.+14 sh.; sta. } 26.1,1 \text { sp. }+3 \text { sh.; sta. } 26.2 \text {, } 1 \text { sp.; sta. } \\
26.3,2 \text { sh.; sta. } 26.4,2 \text { sp. }+1 \text { sh.; sta. } 27.2 \text {, } 1 \text { sp.; sta. } \\
27.3,2 \text { sh.; sta. } 27.4,1 \text { sh.; sta. } 27.5,1 \text { sh.; sta. } 32.5,1 \\
\text { sp.; sta. } 39.1,1 \text { sp. }\end{array}$ \\
\hline Alvania scabra & $6 \mathrm{spc}$. & $\mathrm{Pp}, \mathrm{R}$ & $0.5-1$ & $\begin{array}{l}\text { Sta. } 13.1,2 \text { sp.; sta. } 15.2,1 \text { sp.; sta. } 30.1,1 \text { sp.; sta. } \\
\text { 35.1, } 1 \text { sp.; sta. } 36.2 \text {, } 1 \text { sp. }\end{array}$ \\
\hline Alvania testae & 11 spc., 2 sh. & M & $93-875$ & Sta. 2,8 sp.; sta. 6,3 sp.; sta. 8,2 sh. \\
\hline Alvania marmarisensis n. sp. & $20 \mathrm{spc}$ & M & 100 & Sta. 39.1, $20 \mathrm{sp}$ \\
\hline
\end{tabular}




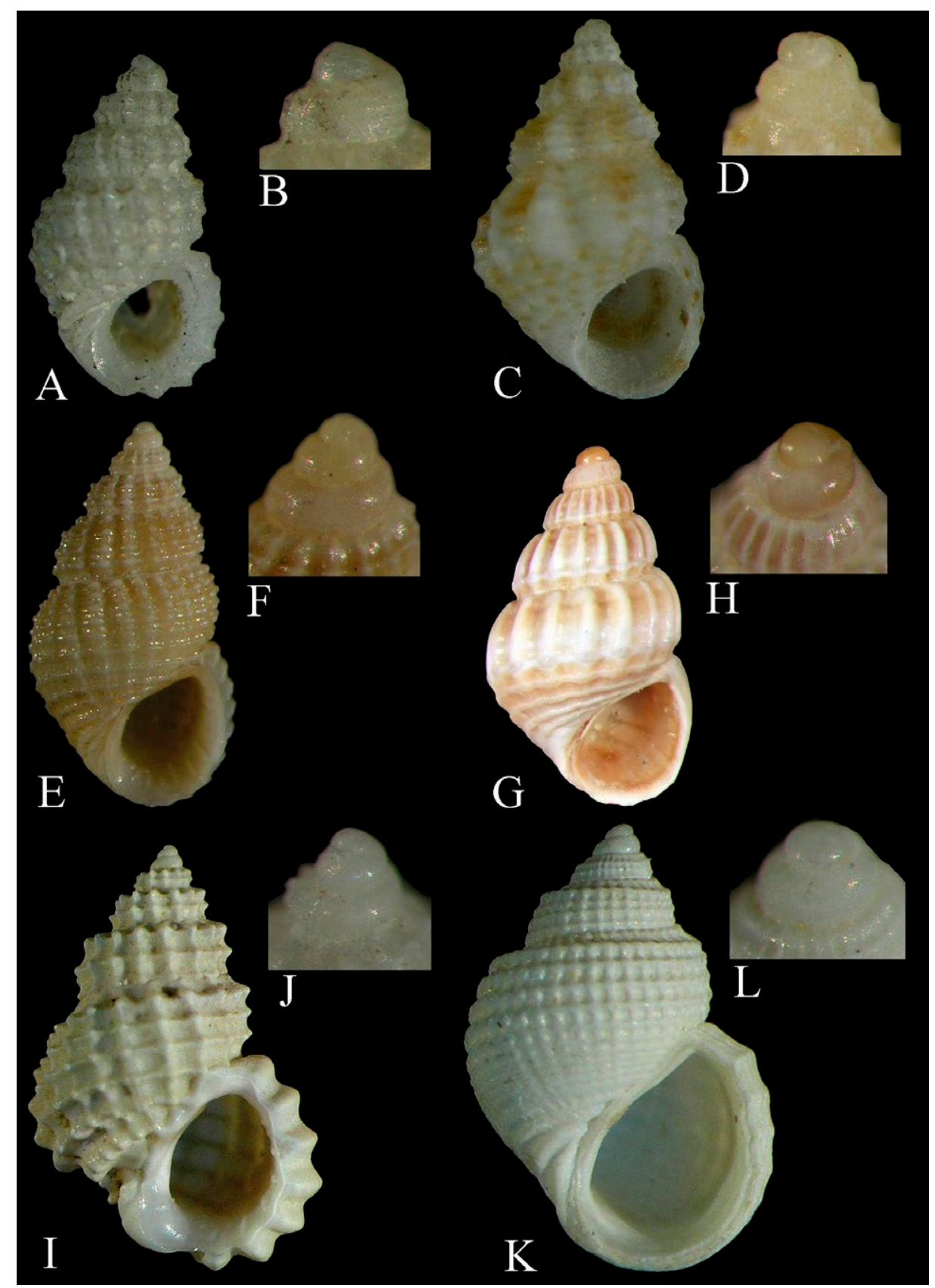

Fig. 2. - Frontal views and protoconchs of some Alvania species distributed in the Aegean Sea. A, B: A. amatii (H=2.6 mm); C, D: A. aspera $(\mathrm{H}=3.2 \mathrm{~mm})$; E, F: A. beanii $(\mathrm{H}=3.1 \mathrm{~mm}) ; \mathrm{G}, \mathrm{H}$ : A. bozcaadensis $(\mathrm{H}=2.4 \mathrm{~mm})$; I, J: A. cancellata $(\mathrm{H}=4.7 \mathrm{~mm})$; and $\mathrm{K}, \mathrm{L}:$ A. carinata $(\mathrm{H}=3.2 \mathrm{~mm})$.

conch, while A. bozcaadensis has a blunt paucispiral apex and fewer axial ribs on the last whorl.

Distribution. Turkish coasts: Levantine and Aegean coasts (Tisselli and Giunchi 2013).

\section{Alvania cancellata (da Costa, 1778)}

(Fig. 2I, J)

Turbo cancellatus da Costa, 1778

Mean dimensions (26 specimens and 5 shells): $3.59( \pm 0.17) \times 2.39$ $( \pm 0.09)-2.57( \pm 0.11) \mathrm{mm}[0.9 \times 0.8-0.7 \mathrm{~mm} ; 4.9 \times 3.3-3.5 \mathrm{~mm}]$.

Remarks. Alvania cancellata is one of the most common and characteristic species of the genus, be- cause of its typical shell structure, large nodule on the base of the columella and multispiral protoconch.

Distribution. Northeast Atlantic Ocean and Mediterranean Sea (Fretter and Graham 1978, Gofas 2007). Turkish coasts: Levantine and Aegean coasts (Demir 2003), Sea of Marmara (Ostroumoff 1894, 1896, Aslan-Cihangir and Ovalis 2013).

\section{Alvania carinata (da Costa, 1778)}

(Fig. 2K, L)

Turbo carinatus da Costa, 1778

Dimensions of two specimens: $2.9 \times 1.9-2.2,3.4 \times 2.4-2.6 \mathrm{~mm}$. 


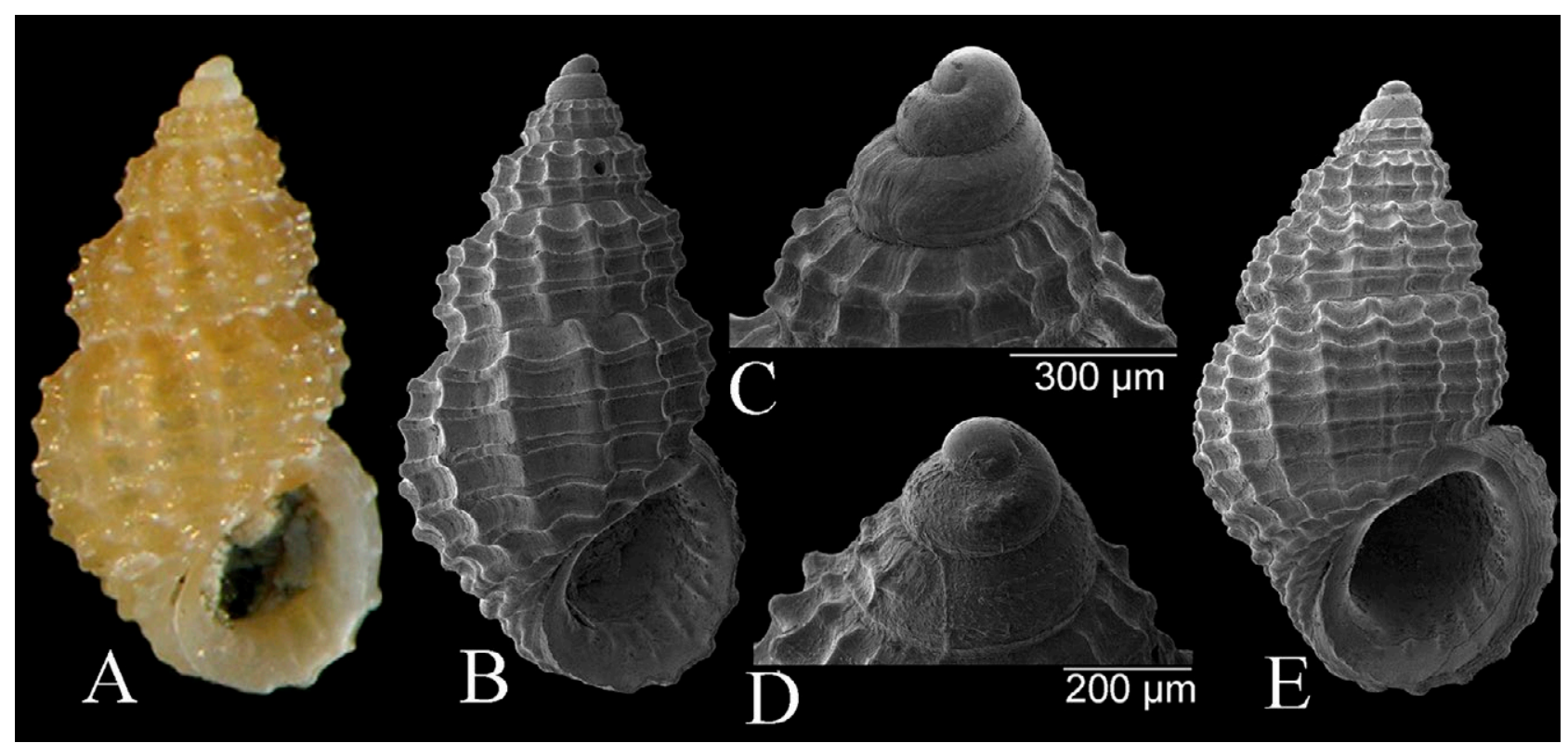

Fig. 3. - A-C, Alvania hispidula, frontal view of a specimen and its protoconch (H=3.4 mm); D, E, Alvania beanii, frontal view of a specimen and its protoconch $(\mathrm{H}=3.1 \mathrm{~mm})$.

Remarks. Alvania carinata can be easily distinguished by the strong spiral sculpture of its teleoconch and paucispiral protoconch.

Distribution. Arctic Ocean (Hudenbick and Warén 1969), northeast Atlantic Ocean and Mediterranean Sea (van Aartsen 1982b). Turkish coasts: Levantine and Aegean coasts (Demir 2003).

\section{Alvania cimex (Linnaeus, 1758)}

(Fig. 4A, B)

Turbo cimex Linnaeus, 1758

Mean dimensions (31 specimens and 35 shells): $3.44( \pm 0.15) \times 2.37$ $( \pm 0.06)-2.58( \pm 0.09) \mathrm{mm}[1.4 \times 1.3-1.2 \mathrm{~mm} ; 5.7 \times 3.4-4.0 \mathrm{~mm}]$.

Remarks. A. cimex is quite similar to A. mamillata, but they differ in the number of whorls of their protoconchs. The protoconch of Alvania cimex consists of 2-2.3 whorls, compared with about 1.3 whorls in $A$. mamillata. However, the protoconch figure given by Gianuzzi-Savelli et al. (1997: 100, fig. 395a) for $A$. cimex does not seem to belong to this species.

It is stated that the number of protoconch whorls was used as a discriminating feature of the shells, but according to some studies (Oliver et al. 2015, Criscione et al. 2016) the protoconch features do not suffice for correct identification. Oliver et al. (2015) considered that $A$. cimex and $A$. mamillata may be the same species because they have the same distribution, habitats and colour patterns, except for the larval life span. Similarly, Criscione et al. (2016: Fig. 2) stated that the two species shared the same $16 \mathrm{~S}$ and $28 \mathrm{~S}$ sequences in the maximum-likelihood phylogram. In this study, we have considered the two species as different taxa, following the current WoRMS (2016) and CLEMAM (2016) databases. Future genetic studies on the larval development of these two species should clarify this aspect.
Distribution. Northeast Atlantic Ocean and Mediterranean Sea (Barash and Danin 1992). Turkish coasts: Levantine Sea (Buzzurro and Greppi 1996, Bitlis Bakır et al. 2012), Aegean Sea (Kocataş 1978), Sea of Marmara (Oberling 1969-1971) and Black Sea (Öztürk 1998).

Alvania cimicoides (Forbes, 1844)

(Fig. 4C, D)

Rissoa cimicoides Forbes, 1844

Mean dimensions (3 specimens and 11 shells): $2.32( \pm 0.21) \times 1.46$ $( \pm 0.10)-1.57( \pm 0.12) \mathrm{mm}[1.2 \times 0.9-0.9 \mathrm{~mm} ; 3.5 \times 2.0-2.3 \mathrm{~mm}]$.

Remarks. This species is characterized by its dark brown-coloured and multispiral protoconch. It is distributed mostly at bathyal depths.

Distribution. Arctic Ocean (Hudenbick and Warén 1969), eastern Atlantic Ocean and Mediterranean Sea (van Aartsen 1982a, Gofas 2007). Turkish coasts: Aegean and Levantine coasts (Forbes 1844, Demir 2003), Sea of Marmara (Ostroumoff 1896, Aslan-Cihangir and Ovalis 2013) and Black Sea (Bacescu et al. 1971).

\section{Alvania colossophilus Oberling, 1970} (Fig. 4E, F)

Alvania colossophilus Oberling, 1970

Dimension (one shell): $3.7 \times 2.2-2.3 \mathrm{~mm}$.

Remarks. A. colossophilus may be confused with $A$. lineata, but the first species differs in the sculpture of its paucispiral protoconch and the stronger and larger size of the shell. 


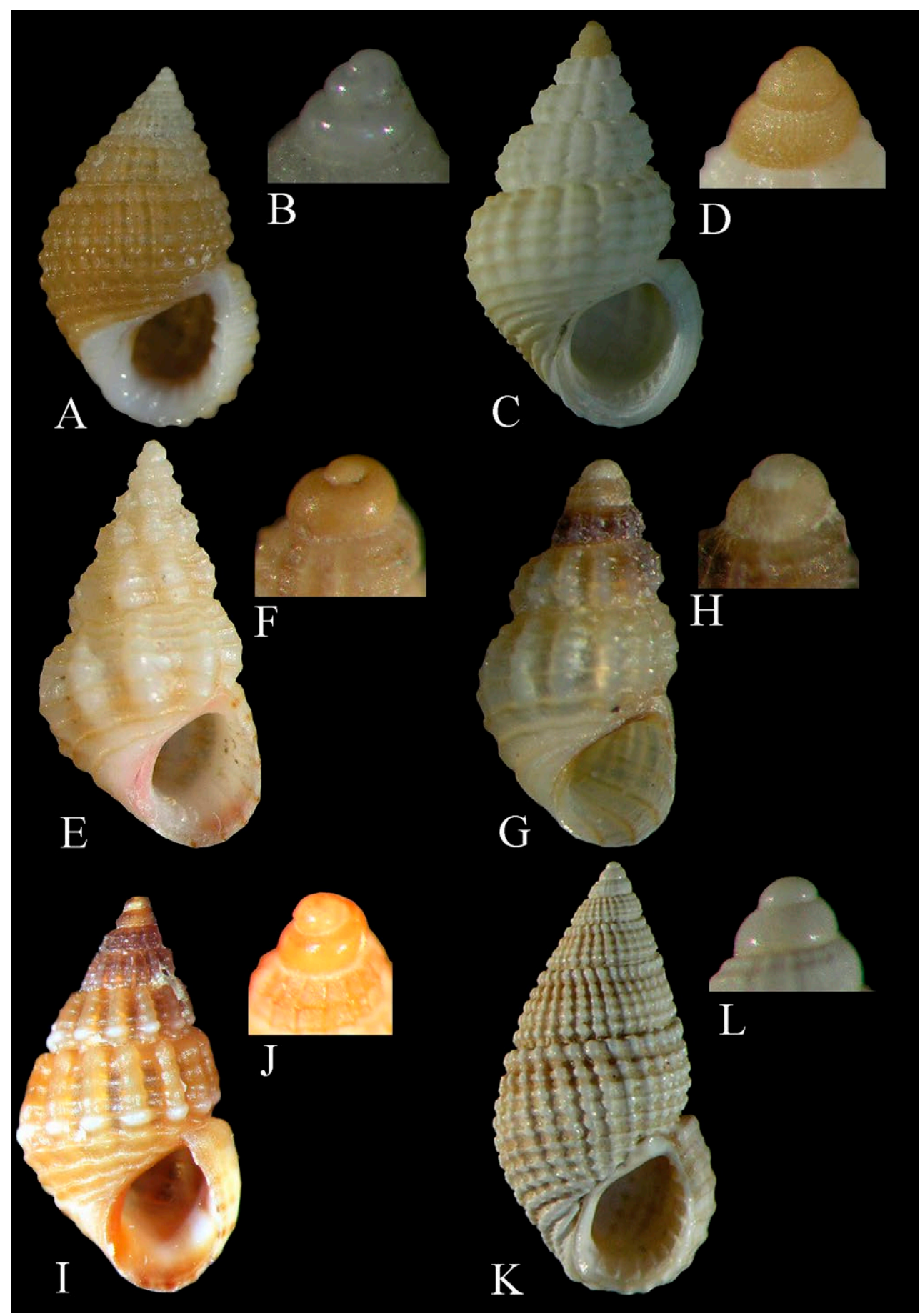

Fig. 4. - Frontal views and protoconchs of some Alvania species distributed in the Aegean Sea. A, B: A. cimex (H=5.0 mm); C, D: A. cimicoides $(\mathrm{H}=3.0 \mathrm{~mm})$; E, F: A. colossophilus $(\mathrm{H}=5.0 \mathrm{~mm}) ; \mathrm{G}, \mathrm{H}:$ A. datchaensis $(\mathrm{H}=2.4 \mathrm{~mm}) ; \mathrm{I}$, $\mathrm{J}$ : A. discors $(\mathrm{H}=4.1 \mathrm{~mm}$, protoconch $\mathrm{H}=3.8$ $\mathrm{mm})$; K, L: A. geryonia $(\mathrm{H}=4 \mathrm{~mm})$.

Distribution. The species appears to be an eastern Mediterranean endemic (Bogi et al. 1989, GianuzziSavelli et al. 1997). Turkish coasts: Levantine Sea (Buzzurro and Greppi 1996, Bitlis Bakır et al. 2012) and Aegean Sea (Okuş et al. 2006).

Alvania datchaensis Amati and Oliverio, 1987 (Fig. 4G, H)

Alvania datchaensis Amati and Oliverio, 1987
Mean dimensions (52 specimens and 17 shells): $2.23( \pm 0.05) \times 1.31$ $( \pm 0.02)-1.53( \pm 0.03) \mathrm{mm}[1.0 \times 0.8-0.8 \mathrm{~mm} ; 3.0 \times 1.8-2.0 \mathrm{~mm}]$.

Remarks. It can be distinguished from the other similar Alvania species by the sculpture of its paucispiral protoconch with zigzag spiral lines, and the outer lip without labial varix.

Distribution. It is endemic to the eastern Mediterranean Sea (Cecalupo and Quadri 1996, Amati 2012). 
Turkish coasts: Levantine Sea (Buzzurro and Greppi 1996) and Aegean Sea (Amati and Oliverio 1987, van Aartsen and Kinzelbach 1990).

\section{Alvania discors (Allan, 1818)}

(Fig. 4I, J)

\section{Turbo discors Allan, 1818}

Mean dimensions (218 specimens and 82 shells): $3.12( \pm 0.09) \times$ $1.97( \pm 0.03)-2.13( \pm 0.05) \mathrm{mm}[1.1 \times 0.9-0.8 \mathrm{~mm} ; 5.3 \times 3.0-3.4$ $\mathrm{mm}]$.

Remarks. The species is characterized by its polymorphic shell with a variable number of axial ribs (1112 ) and spiral cords (8-9) on the last whorl. The protoconch is multispiral, and its shell may be completely brownish or with white bands.

Distribution. Northeast Atlantic Ocean and Mediterranean Sea (Barash and Danin 1992, Cecalupo and Quadri 1996). Turkish coasts: Levantine Sea (Buzzurro and Greppi 1996, Bitlis Bakır et al. 2012), Aegean Sea (Kocataş 1978) and Sea of Marmara (Oberling 1969-1971).

Alvania geryonia (Nardo, 1847) (Figs 4K, L, 7J)

Rissoa geryonia Nardo, 1847

Mean dimensions (26 specimens and 24 shells): $3.29( \pm 0.10) \times 1.91$ $( \pm 0.04)-2.27( \pm 0.06) \mathrm{mm}[1.1 \times 0.8-0.8 \mathrm{~mm} ; 4.5 \times 3.3-3.5 \mathrm{~mm}]$.

Remarks. Alvania geryonia can be misidentified with $A$. cimex, but the first one has more spirals on the penultimate whorl (5 spiral cords contrary to 3 spiral cords in A. cimex) and smaller granules on the teleoconch. The protoconch is multispiral.

Distribution. Northeast Atlantic Ocean and Mediterranean Sea (van Aartsen 1982a, Gofas et al. 2011). Turkish coasts: Levantine Sea (Demir 2003, Bitlis Bakır et al. 2012), Aegean Sea (Demir 2003, Çınar et al. 2012) and Sea of Marmara (Ostroumoff 1896, Demir 2003).

\section{Alvania hispidula (Monterosato, 1884)}

(Fig. 3A-C)

\section{Acinus hispidulus Monterosato, 1884}

The shell is solid with 4 convex teleoconch whorls and deep suture. Protoconch is multispiral. On the last whorl, above the aperture, there are 10-14 axial ribs, and 3 spiral cords on the last and penultimate whorls. There is a noteworthy distance between the first spiral cords and the suture. Thickened labrum and light brown coloured shell.

Mean dimensions $(2$ specimens and 4 shells $): 3.42( \pm 0.09) \times 1.72$ $( \pm 0.10)-2.19( \pm 0.06) \mathrm{mm}[3.0 \times 1.3-1.9 \mathrm{~mm} ; 3.7 \times 1.9-2.3 \mathrm{~mm}]$.

Remarks. Alvania hispidula is similar to A. beanii but differs in the number of spiral and axial ribs on the last whorl. Alvania beanii has a smaller shell and 6-7 spiral cords on the last whorl above the aperture, instead of 3 spirals in A. hispidula (4 in the penultimate whorl). The protoconch of A. hispidula (Fig. 3C) and
A. beanii (Fig. 3D) are similar, but the protoconch of $A$. beanii has micro papillae.

Distribution. Atlantic Ocean and Mediterranean Sea (van Aartsen 1982a). Turkish coasts: Sea of Marmara (Ostroumoff 1896) and Aegean Sea (this study).

\section{Alvania lactea (Michaud, 1832)}

(Fig. 5A, B)

Rissoa lactea Michaud, 1832

Mean dimensions $(11$ specimens and 6 shells $): 3.25( \pm 0.24) \times 2.20$ $( \pm 0.12)-2.69( \pm 0.19) \mathrm{mm}[1.2 \times 1.1-1.1 \mathrm{~mm} ; 4.8 \times 3.0-3.8 \mathrm{~mm}]$.

Remarks. A. lactea differs markedly from the other species of the genus in its shell sculpture and large aperture. The protoconch is paucispiral.

Distribution. Arctic Ocean (Hudenbick and Warén 1969), North Atlantic Ocean and Mediterranean Sea (Barash and Danin 1992). Turkish coasts: Levantine Sea (Buzzurro and Greppi 1996, Bitlis Bakır et al. 2012), Aegean Sea (Demir 2003) and Sea of Marmara (Oberling 1960-1962, 1969-1971).

\section{Alvania lineata Risso, 1826}

(Fig. 5C, D)

\section{Alvania costulosa Risso, 1826}

Mean dimensions (4 specimens): $3.22( \pm 0.16) \times 2.07( \pm 0.10)-2.27$ $( \pm 0.13) \mathrm{mm}[2.8 \times 1.9-2.0 \mathrm{~mm} ; 3.6 \times 2.3-2.6 \mathrm{~mm}]$.

Remarks. The species differs from the other species of the genus in its large paucispiral protoconch ornamented with spiral threads and in its strong shell, with its axial ribs as wide as the interspaces, with prominent spirals.

Distribution. Mediterranean Sea (Gofas et al. 2011). Turkish coasts: Aegean Sea (Kocataş 1978, Demir 2003), Levantine Sea (Demir 2003) and Dardanelles (Aslan-Cihangir and Ovalis 2013).

Alvania mamillata Risso, 1826

(Figs 5E, F, 6G, 7F-I)

Alvania mamillata Risso, 1826

Mean dimensions (32 specimens and 23 shells): $3.64( \pm 0.13) \times 2.42$ $( \pm 0.06)-2.77( \pm 0.08) \mathrm{mm}[1.4 \times 1.2-1.1 \mathrm{~mm} ; 4.9 \times 3.2-3.7 \mathrm{~mm}]$.

Remarks. (See remarks for A. cimex). The paucispiral protoconch (about 1.3 whorls) and coarse granules on the teleoconch of A. mamillata are noteworthy features of the species.

Distribution. Northeast Atlantic Ocean and Mediterranean Sea (Zenetos and van Aartsen 1995). Turkish coasts: Levantine Sea (Bitlis Bakır et al. 2012) and Aegean Sea (van Aartsen and Kinzelbach 1990).

Alvania punctura (Montagu, 1803) (Fig. 5G, H) 


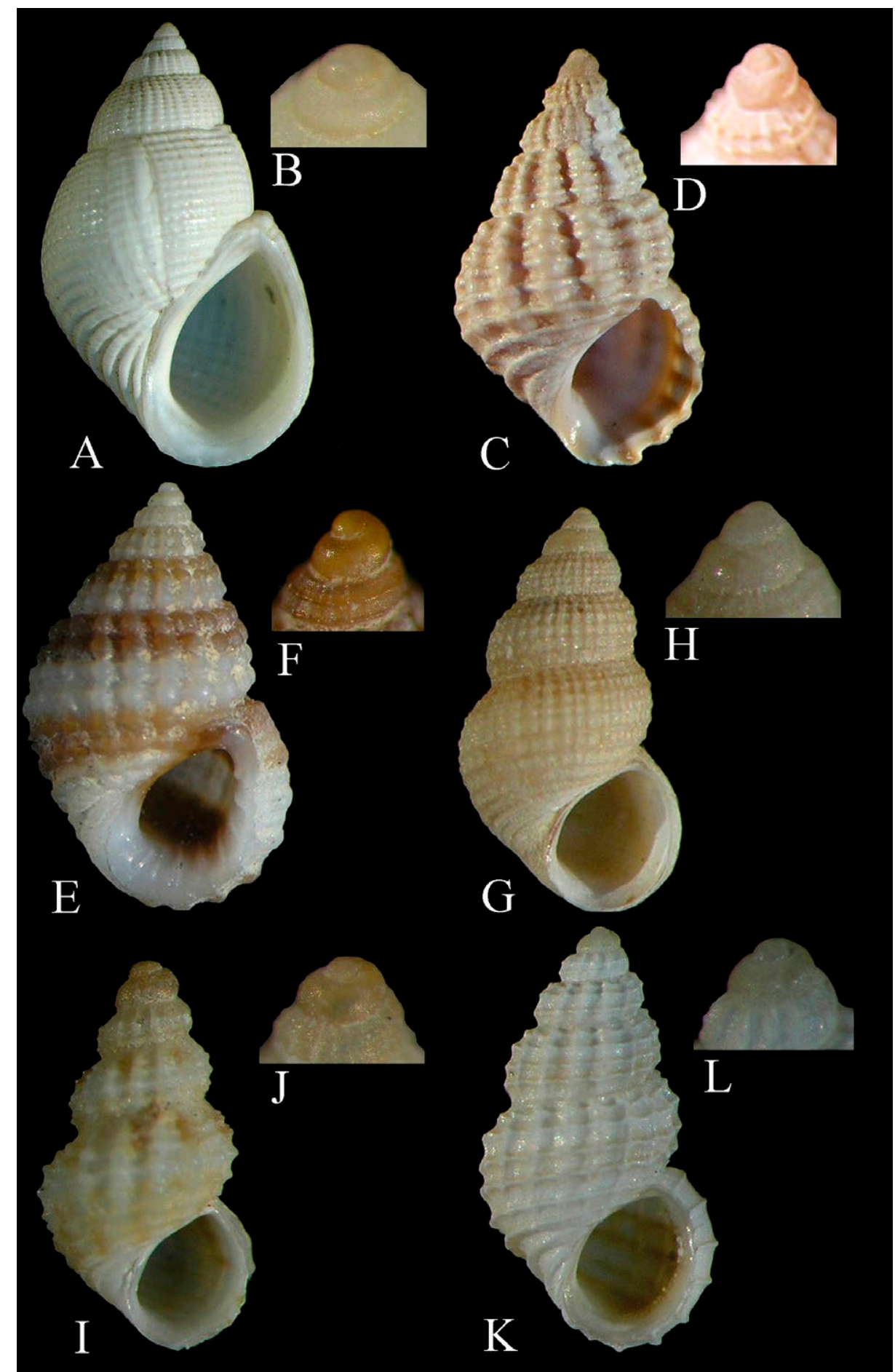

Fig. 5. - Frontal views and protoconchs of some Alvania species distributed in the Aegean Sea. A, B: A. lactea $(\mathrm{H}=5.0 \mathrm{~mm})$; C, D: A. lineata $(\mathrm{H}=3.6 \mathrm{~mm}$, protoconch $\mathrm{H}=2.8 \mathrm{~mm}) ; \mathrm{E}, \mathrm{F}:$ A. mamillata $(\mathrm{H}=4.6 \mathrm{~mm}) ; \mathrm{G}, \mathrm{H}:$ A. punctura $(\mathrm{H}=2.9 \mathrm{~mm}) ; \mathrm{I}, \mathrm{J}:$ A. scabra $(\mathrm{H}=2.1 \mathrm{~mm}) ; \mathrm{K}, \mathrm{L}:$ A. testae $(\mathrm{H}=2.5 \mathrm{~mm})$.

Turbo punctura Montagu, 1803

Mean dimensions (42 specimens and 24 shells): $1.78( \pm 0.05) \times 1.06$ $( \pm 0.02)-1.21( \pm 0.02) \mathrm{mm}[1.1 \times 0.8-0.8 \mathrm{~mm} ; 2.5 \times 1.3-1.5 \mathrm{~mm}]$.

Remarks. It is recognizable by its multispiral protoconch and clathrate sculpture of the teleoconch.

Distribution. Arctic Ocean (Hudenbick and Warén 1969), Atlantic Ocean and Mediterranean Sea (van
Aartsen 1982c, Gofas 2007). Turkish coasts: Aegean Sea (Demir 2003) and Sea of Marmara (Ostroumoff 1896).

Alvania scabra (Philippi, 1844)

(Fig. 5I, J)

Rissoa scabra Philippi, 1844

Mean dimensions (6 specimens): $1.70( \pm 0.11) \times 1.01( \pm 0.05)-1.12$ 


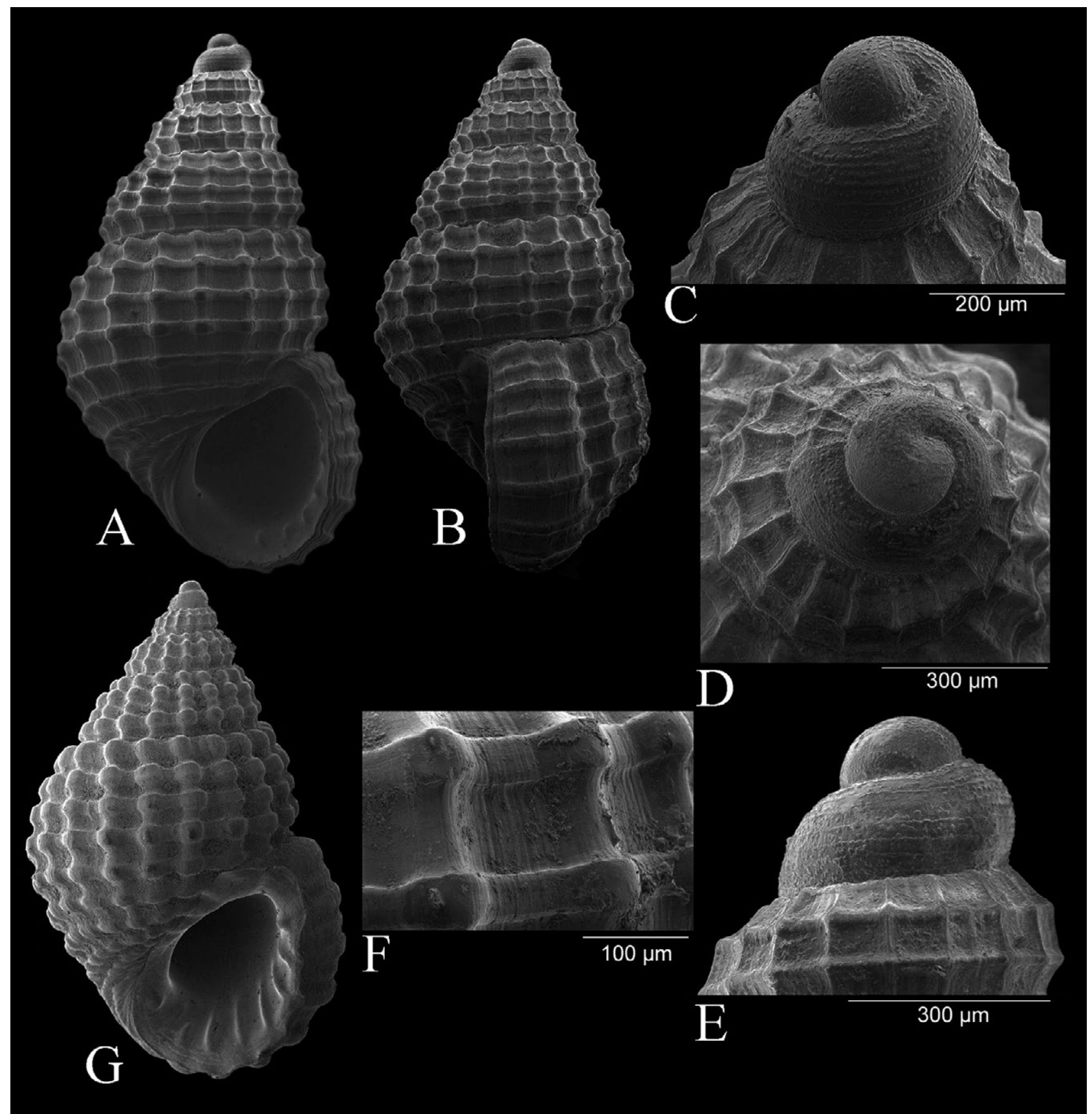

Fig. 6. - A-F: Alvania marmarisensis n. sp., frontal and lateral views of the holotype and its protoconch (C, D, E), and microsculpture on the teleoconch whorls $(\mathrm{F})(\mathrm{H}=3.3 \mathrm{~mm}, \mathrm{~W}=1.9 \mathrm{~mm}, \mathrm{LWH}=2.2 \mathrm{~mm}$, Marmaris, Turkey, $99 \mathrm{~m}$. G: Alvania mamillata, frontal view of a specimen $(\mathrm{H}=4.6 \mathrm{~mm})$.

$( \pm 0.05) \mathrm{mm}[1.4 \times 0.8-1.0 \mathrm{~mm} ; 2.1 \times 1.2-1.3 \mathrm{~mm}]$.

Remarks. Alvania scabra differs from similar Alvania species in having more spiral cords on the penultimate 4 and last whorls 7-8, respectively. The protoconch is paucispiral.

There are uncertainties about the synonyms of $A$. scabra. According to the Checklist of European Marine Mollusca (CLEMAM 2016), A. oranica was regarded as a synonym of A. scabra. van Aartsen (1982c) examined this idea and remarked that the mean difference between $A$. scabra and A. oranica consisted in having a fourth spiral (always) in the subsutural area of A. oranica, which is rarely found in A. scabra. In addi- tion, Tringali (2001) indicated that the original description and figure of $A$. oranica given by Pallary (1900: 322, pl. 7, Fig. 4) was inadequate and still unclear and, due to this fact, $A$. oranica might be a synonym of A. sculptilis but not of A. scabra. Tringali (2001) also underlined that the figure of $A$. oranica provided by Gofas (1990: 130, Fig. 58) did not belong to $A$. oranica. He also stated that the figure of A. scabra in the study by van Aartsen et al. (1984) belongs to $A$. sculptilis (Monterosato, 1877) instead of A. scabra. In this study, we agree with Tringali's opinion about the original description of $A$. oranica, which is insufficient and requires improvement. 
Distribution. Mediterranean Sea (van Aartsen 1982c, Gofas et al. 2011). Turkish coasts: Levantine Sea (Buzzurro and Greppi 1996) and Aegean Sea (van Aartsen and Kinzelbach 1990).

Alvania testae (Aradas and Maggiore, 1844) (Fig. 5K, L)

Rissoa testae Aradas and Maggiore, 1844

Mean dimensions $(11$ specimens and 2 shells $): 2.26( \pm 0.05) \times 1.22$ $( \pm 0.03)-1.42( \pm 0.03) \mathrm{mm}[1.9 \times 1.0-1.2 \mathrm{~mm} ; 2.5 \times 1.4-1.5 \mathrm{~mm}]$.

Remarks. Alvania testae is characterized by its shell shape. The shell is narrow and conical with 5-6 convex teleoconch whorls. Its protoconch is paucispiral. Aperture is roundish and outer lip opisthocline.

Distribution. Atlantic Ocean and Mediterranean Sea (Hoenselaar and Goud 1998, Gofas et al. 2011). Turkish coasts: Levantine Sea (Bitlis Bak1r et al. 2012), Aegean Sea (Demir 2003, Öztürk et al. 2008) and Sea of Marmara (Ostroumoff 1896, Demir 2003).

\section{Alvania marmarisensis n. sp.} (Figs 6A-F, 7A-E)

Type material and type locality: Holotype (ESFM-GAS/2014-10): Marmaris, 99 m, Aegean Sea; H: $3.3 \mathrm{~mm}$, W: $1.9 \mathrm{~mm}, \mathrm{BWH}: 2.2$ mm. Paratypes: 11 specimens (ESFM-GAS/2014-10).

Mean dimensions $(20$ specimens $): 2.96( \pm 0.08) \times 1.84( \pm 0.03)-$ $2.07( \pm 0.04) \mathrm{mm}[2.3 \times 1.6-1.7 ; 3.5 \times 2.1-2.4 \mathrm{~mm}]$.

Etymology. The name of the species is derived from the name of the area (Marmaris) where the specimens were found.

Description. Shell (holotype): Conical in shape, not very solid, with 4.5 less convex teleoconch whorls. Protoconch (holotype) is paucispiral ( 1.25 whorls) and its nucleus diameter is about $0.150 \mathrm{~mm}$. It has irregular spiral lines with rounded micro papillae in the interspaces.

Teleoconch (holotype) has four spirals on the penultimate whorl, 15 axial ribs in the last whorl, 4 spiral cords above the aperture, and 5 spirals on the base. The suture is deep, with a slope between the first and second spiral cords in the penultimate and last whorls. It has small granules at the intersections of the axial ribs, with spiral cords on the teleoconch whorls. There are also fine growth lines on the teleoconch. The aperture is large and ovate. The outer lip is thickened, with 7 internal denticles. It is light-brown coloured, while the apex is often darker. The soft parts and operculum were not examined.

Remarks. Among the species distributed along the Turkish Aegean coast, A. marmarisensis has some similarities with A. beanii, A. geryonia and A. mamillata (Fig. 7). However, it differs from $A$. beanii and A. geryonia in the number of protoconch whorls and rounded irregular micro papillae. The teleoconch whorls of $A$. beanii are more convex and have a greater number of axial ribs (24-26) and spiral cords (6-7). Alvania geryonia has a differently shaped teleoconch, with nearly flat whorls, and 5 spirals on the penultimate whorl. The protoconch of A. marmarisensis is also similar to that of A. mamillata (Verduin 1986: 28, Fig. 3; Oliver et al. 2015: 114, Figs 9, 10, 12, 13), but differs from A. mamillata in being more rounded and having large micro papillae on the protoconch whorls. On the other hand, A. mamillata has a more solid shell and a higher number of axial ribs on the last teleoconch whorl (14-20), and coarser granules at the intersections of the axial ribs with the spirals.

Alvania marmarisensis $\mathrm{n}$. sp. is also morphologically similar to some other Mediterranean species, (i.e. Alvania dalmatica Buzzurro and Prkic, 2007, Alvania dianiensis Oliverio, 1988, Alvania hallgassi Amati and Oliverio, 1985 and Alvania oliverioi Buzzurro, 2003). The new species differs from Alvania dalmatica, A. dianiensis, A. hallgassi and A. oliverioi in the features of its protoconch and teleoconch, geographic distribution and depth ranges (Table 3). Alvania marmarisensis has 1.15-1.25 protoconch whorls, which are ornamented with 5-6 interrupted spiral threads with micro papillae in the interspaces, whereas A. dalmatica has 1.3-1.5 protoconch whorls bearing 6-7 spiral threads of the same thickness, with no papillae in the interspaces (Buzzurro and Prkic 2007: 6, Figs b, c). In addition, the new species has more spiral cords on the penultimate whorl (4 spirals against 3 in A. dalmatica) and on the last whorl (8-9 spirals against 5-9 spirals in $A$. dalmatica), but the number of denticles inside the outer lip of A. marmarisensis is smaller (6-7) than that of $A$. dalmatica (7-11). The colour of A. marmarisensis is light brown without coloured stripes as in A. dalmatica.

A. marmarisensis differs from A. hallgassi in having fewer axial ribs on the last whorl (13-17 against 15-40) and no coloured stripes on the whorls. Apart from the differences in the teleoconch, A. marmarisensis has a lower number of protoconch whorls (1.15-1.25 against 1.5 in A. hallgassi) bearing micro papillae in the interspaces between the spirals, which are smooth in A. hallgassi (Amati and Oliverio 1985: 34, Figs 1, 2).

Alvania marmarisensis also differs from A. oliverioi in having a higher teleoconch $(\mathrm{h}=2.3-3.5 \mathrm{~mm}$ against $\mathrm{h}=1.54 \mathrm{~mm}$ ) and a small number of axial ribs on the last whorl (13-17 against 39-40). The protoconch of $A$. oliverioi consists of 1.4 whorls with 4-5 weak spirals and regular micro papillae on the whorls (Buzzurro 2003: 44-45, Figs 2-4) (Table 3).

The protoconch of $A$. marmarisensis is similar to that of $A$. dianiensis, except for the protoconch whorls (1.5) (Oliverio 1988: 120, Figs 2-7). Alvania marmarisensis is also larger $(\mathrm{h}=2.3-3.5 \mathrm{~mm})$ and has a higher number of teleoconch whorls (4-4.5) than A. dianiensis $(\mathrm{h}=2-2.4 \mathrm{~mm}$ and 3.2 whorls). Alvania marmarisensis also bears a higher number of spiral cords on the last whorl (8-9 against 6-7), and 4 spirals against 3 spirals above the aperture. There are four spirals on the penultimate whorl in A. marmarisensis against 3 spirals in $A$. dianiensis. The other difference between the aforementioned two species regards the microsculpture of the teleoconchs; A. marmarisensis has only fine growth lines contrary to fine spiral striae in A. dianiensis (Oliverio 1988, Romani 2014). 


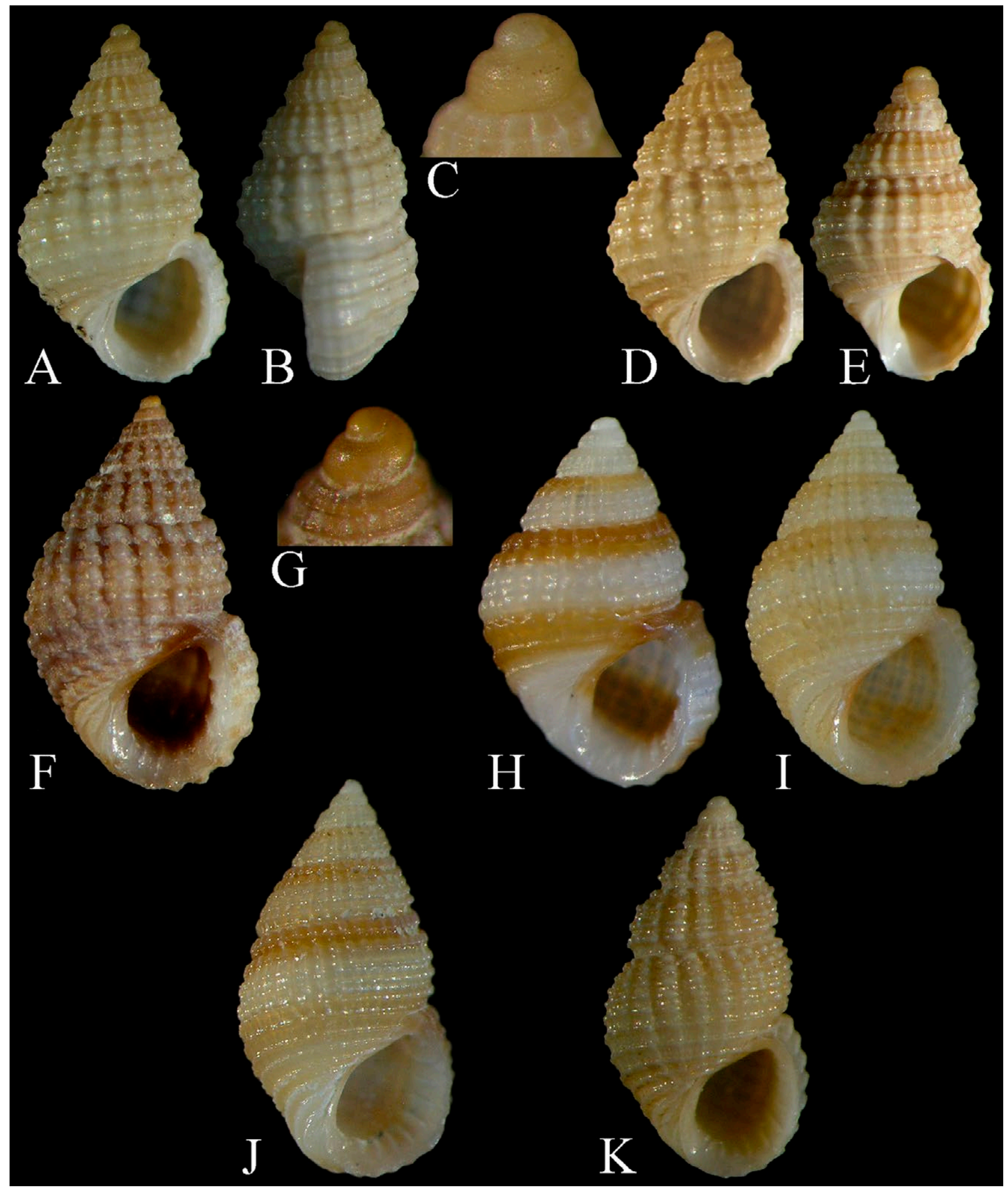

Fig. 7. - A-E, Alvania marmarisensis n. sp., frontal and lateral views of the holotype and its protoconch $(\mathrm{C})(\mathrm{H}=3.3 \mathrm{~mm})$; D: frontal view of the paratype $(\mathrm{H}=3.9 \mathrm{~mm})$; E: frontal view of a juvenil $(\mathrm{H}=2.6 \mathrm{~mm}) ; \mathrm{F}, \mathrm{G}$ : Alvania mamillata, frontal view of a specimen and its protoconch $(\mathrm{H}=4.4 \mathrm{~mm}) ; \mathrm{H}$ : Alvania mamillata $(\mathrm{H}=3.6 \mathrm{~mm})$; I: Alvania mamillata $(\mathrm{H}=4.5 \mathrm{~mm}) ; \mathrm{J}$ : Alvania geryonia $(\mathrm{H}=3.9 \mathrm{~mm}) ; \mathrm{K}:$ Alvania beanii $(\mathrm{H}=3.1 \mathrm{~mm})$.

There are also differences in the distribution areas and bathymetric ranges among A. marmarisensis and similar species (Table 3 ). With the exception of $A$. marmarisensis and A. dalmatica, all of the mentioned species were collected from infralittoral depths. Alvania marmarisensis was found at a depth of $99 \mathrm{~m}$ along the Aegean Sea coast (Marmaris) and Alvania dalmatica was recorded from the coasts of Croatia, Greece and the Adriatic Sea at depths ranging from 40 to $90 \mathrm{~m}$ (Buzzurro and Prkic 2007, Romani 2014). As regards the species that are similar to A. marmarisensis, A. dianiensis was reported from the Tyrrhenian, Ligurian 
Table 3. - Some characteristics of protoconch and teleoconch, bathymetric ranges, and distribution of the Alvania species similar to Alvania marmarisensis n. sp. (H, total height of teleoconch; Nw, number of teleoconch whorls; Nar, number of axial ribs on the last whorl; Nsc, number of spiral cords on the last whorl; Nsca, number of spiral cords above the aperture; Nscp, number of spiral cords on the penultimate whorl; Npw, number of protoconch whorls; Psc, protoconch sculpture).

\begin{tabular}{|c|c|c|c|c|c|}
\hline & A. marmarisensis & A. dalmatica & A. dianiensis & A. hallgassi & A. oliverioi \\
\hline $\mathrm{H}$ & $2.3-3.5$ & $2.3-3.5$ & $2-2.4$ & $2-3$ & 1.54 \\
\hline Nw & $4-4.5$ & $3.1-4$ & 3.2 & 3 & $2.4-2.5$ \\
\hline Nar & $13-17$ & $13-22$ & $14-23$ & $15-40$ & $39-40$ \\
\hline Nsc & $8-9$ & $5-9$ & $6-7$ & $8-11$ & 10 \\
\hline Nsca & 4 & $2-5$ & 3 & $4-7$ & 5 \\
\hline Nscp & 4 & 3 & 3 & $3-4$ & 3 \\
\hline Denticles & $6-7$ & $7-11$ & No & No & No \\
\hline Npw & $1.15-1.25$ & $1.3-1.5$ & 1.5 & 1.5 & 1.4 \\
\hline Psc & $\begin{array}{l}5-6 \text { spiral threads, with } \\
\text { micro papillae }\end{array}$ & $\begin{array}{l}\text { 6-7 spiral threads, with } \\
\text { no micro papillae }\end{array}$ & $\begin{array}{l}5-6 \text { spiral threads, with } \\
\text { micro papillae }\end{array}$ & $\begin{array}{l}\text { 5-6 spiral threads, with } \\
\text { no micro papillae }\end{array}$ & $\begin{array}{l}4-5 \text { weak spirals, with } \\
\text { micro papillae }\end{array}$ \\
\hline Colour & Light brown, apex dark & $\begin{array}{l}\text { Light yellow, with two } \\
\text { stripes }\end{array}$ & Yellowish, whitish & $\begin{array}{l}\text { Yellowish with two } \\
\text { dark stripes }\end{array}$ & $\begin{array}{l}\text { Whitish and light } \\
\text { yellow with two stripes }\end{array}$ \\
\hline Habitat & Mud & $\begin{array}{l}\text { Corallina rubrum, } \\
\text { coralligenous }\end{array}$ & Photophilic algae & Photophilic algae & Detritic mud \\
\hline Depth & $99 \mathrm{~m}$ & $40-90 \mathrm{~m}$ & $18-48 \mathrm{~m}$ & down to- $20 \mathrm{~m}$ & $4 \mathrm{~m}$ \\
\hline Locality & Aegean Sea & $\begin{array}{l}\text { Croatia, Greece, } \\
\text { Adriatic Sea }\end{array}$ & $\begin{array}{l}\text { Tyrrhenian Sea and } \\
\text { Ligurian, Adriatic Sea }\end{array}$ & $\begin{array}{l}\text { Ionian Sea, Sicily, } \\
\text { Adriatic Sea, } \\
\text { Tyrrhenian Sea and } \\
\text { Cyprus }\end{array}$ & Cyprus \\
\hline References & Present study & $\begin{array}{l}\text { Buzzurro and Prkic } \\
(2007) \text {, Romani (2014) }\end{array}$ & Oliverio (1988) & $\begin{array}{l}\text { Amati and Oliverio } \\
\text { (1985), Romani (2014) }\end{array}$ & Buzzurro (2003) \\
\hline Type material & $\begin{array}{l}\text { GAS/2014-10 } \\
\text { Museum of Faculty } \\
\text { of Fisheries at Ege } \\
\text { University (ESFM), } \\
\text { Turkey } \\
\text { Marmaris (Aegean Sea) }\end{array}$ & $\begin{array}{l}\text { MSNM Mo } 31380 \\
\text { Malacological } \\
\text { Collection of Museo } \\
\text { Civico di Storia } \\
\text { Naturale of Milano } \\
\text { (MNHM), Italy } \\
\text { Islands of Mljet and } \\
\text { Susac (Dalmatia, } \\
\text { Croatia) }\end{array}$ & $\begin{array}{l}\text { ZMA Moll. No. } \\
3.88 .019 \\
\text { Zoölogisch Museum } \\
\text { Amsterdam (ZMA) } \\
\text { Isle of Gianutri (Italy) }\end{array}$ & $\begin{array}{l}\text { Coll. Oliverio, Coll. } \\
\text { Amati, Coll. Hallgass } \\
\text { Civico Museo } \\
\text { Zoologico di Roma } \\
\text { Otranto (Puglia) }\end{array}$ & $\begin{array}{l}\text { MNHM Mo } 26541 \\
\text { Malacological } \\
\text { Collection of Museo } \\
\text { Civico di Storia } \\
\text { Naturale of Milano } \\
\text { (MNHM), Italy } \\
\text { Girne (Northern } \\
\text { Cyprus) }\end{array}$ \\
\hline
\end{tabular}

and Adriatic Seas, from algal beds at depths ranging from 18 to $48 \mathrm{~m}$ (Oliverio 1988, Romani 2014), whereas Alvania hallgassi was described from infralittoral depths of the Ionian Sea (down to $20 \mathrm{~m}$ ) (Amati and Oliverio 1985). This last species was also reported from Sicily, the Adriatic Sea, the Tyrrhenian Sea and the coasts of Cyprus (Oliverio et al. 1986, Cecalupo and Quadri 1996, Romani 2014). Alvania oliverioi was found along the Cypriot coastline at a depth of $4 \mathrm{~m}$ (Buzzurro 2003).

\section{DISCUSSION}

Among the analysed benthic material, we sorted 537 living specimens and 249 empty shells belonging to 20 species of the genus Alvania. Of the identified species, Alvania marmarisensis is described as a new species. The new species was only recorded near Marmaris at station 39.1 in muddy material sampled at 99 $m$ depth.

A. mamillata was the commonest species, found at 18 stations in the Aegean Sea, followed by A. discors, found at 15 stations, whereas A. colossophilus was the rarest species with only one specimen found at station 27 (Table 2).

Alvania discors was the most abundant species, with 218 specimens and 82 shells, followed by A. datchaensis (52 specimens and 17 shells). However, A. bozcaadensis, A. carinata and A. colossophilus were only represented by a few specimens or shells (Table 2).

Regarding the bathymetric distribution of the identified species, most of them (14 species) were collected at depths ranging between 0 and $10 \mathrm{~m}$, while 8 species
(A. beanii, A. cancellata, A. cimex, A. datchaensis, A. geryonia, A. hispidula, A. mamillata and A. punctura) were found at depths between 10 and $25 \mathrm{~m}, 6$ species (A. beanii, A. cancellata, A. cimex, A. colossophilus, A. geryonia, A. punctura) at depths between 25 and $50 \mathrm{~m}$, and 7 species (A. beanii, A. cancellata, A. geryonia, A. hispidula, A. punctura, A. testae, A. marmarisensis) at depths between 50 and $100 \mathrm{~m}$. Alvania cimicoides and A. testae were distributed at bathyal depths between 93 and $875 \mathrm{~m}$ (Table 2).

Bouchet and Warén (1993), in their study on the bathyal and abyssal mesogastropod species of the northeastern Atlantic Ocean, dealt with many rissoids, of which 21 species belong to the genus Alvania. Among the Alvania species reported by these authors, A. cimicoides was found at depths between 36 and $4700 \mathrm{~m}$, and A. testae was collected at depths between 36 and $2400 \mathrm{~m}$. In this study, those two species were also found at circalittoral and bathyal depths (93-875 m; sta. 2, sta. 6, sta. 7, sta. 8 and sta. 28).

Bitlis Bakır and Öztürk (2016) investigated Rissooidea species distributed along the Turkish Levantine coast and reported 15 Alvania species, which have also been examined within the context of this study. A. beanii (60-1302 m) and A. testae (100-200 m), which were sampled at circalittoral and bathyal depths in the mentioned study, were encountered in our study at depths between 23 and $100 \mathrm{~m}$ and 93 and $875 \mathrm{~m}$, respectively.

Regarding the habitats where these species were found, seagrass meadows (Posidonia oceanica, Zostera noltei, Z. marina, and Cymodocea nodosa) were the most diverse, housing 14 species, followed by algal beds (Padina pavonica, Cystoseira amentacea, C. crinita, C. 
foeniculacea $f$. schiffneri, C. spinosa, C. elegans, $C$. compressa, Halopteris scoparia, H. filicina, Ulva linza) and hard substrates (rock, Cladocora caespitosa, Aplysina aerophoba, Pinna nobilis), both with 11 species, and soft substrates (sand, mud, mixture of sand and mud, and coralligens) with 10 species (Table 2).

The preferred habitats of Alvania species found during this study are in agreement with those given by Antoniadou et al. (2005), Albano and Sabelli (2012), Pitacco et al. (2014). In the North Aegean Sea, A. aspera, A. cimex, and A. mamillata were reported from habitats of photophilic algae, and A. discors was found in soft sediments and in $P$. oceanica (Antoniadou et al. 2005). In this study, $A$. aspera was encountered in algal beds, while A. cimex, A. discors and A. mamillata were found in photophilic algae, soft sediments and $P$. oceanica. Albano and Sabelli (2012) examined the molluscan fauna inhabiting the leaves and rhizomes of $P$. oceanica distributed in the central Tyrrhenian Sea and reported A. cancellata, A. hispidula and $A$. settepassii. Likewise, A. cancellata and A. hispidula were encountered in $P$. oceanica meadows along the Turkish Aegean coast. Pitacco et al. (2014) stated that A. cimex, A. discors and A. lineata preferred Cystoseira beds in the Gulf of Trieste. In this study, those Alvania species were also found in Cystoseira communities (C. amantacea, C. crinita, C. compressa, C. spinosa, C. foeniculacea f. schiffneri). Poursanidis and Koutsoubas (2015) reported 15 Alvania species inhabiting the coralligenous habitats of the Mediterranean Sea, of which only A. beanii, A. cancellata and A. geryonia were found in this study. In a study carried out in the Mediterranean Sea by Poursanidis et al. (2016), 21 Alvania species were reported from the infralittoral rocky shores and photophilic algae, of which $A$. cimex, A. discors, A. geryonia, A. lactea, A. lineata, A. mamillata, A. scabra were also found during our study, in rocky and algal biotopes. Along the Levantine coast of Turkey, A. mamillata, A. geryonia, A. discors and A. datchaensis were found to be dominant in sandy biotopes, seagrass meadows and red algae (Bitlis Bakır and Öztürk 2016). As regards the species of this study, A. mamillata and A. geryonia were found in soft sediments, phanerogams, hard substrate and brown algae, whereas $A$. discors and $A$. datchaensis were encountered in phanerogam meadows, hard substrates and brown algae.

It is assumed that the difference in the number of protoconch whorls depends on differences in larval development (planktotrophic vs non-planktotrophic), which may affect the distribution range of the species. Therefore, eight Alvania species (A. beanii, A. cancellata, A. cimex, A. cimicoides, A. discors, $A$. geryonia, $A$. hispidula and $A$. punctura) with multispiral protoconch examined during this study have a wide distribution in the Atlantic Ocean and the Mediterranean Sea. However, A. amatii, A. lineata, A. scabra, A. aspera, A. bozcaadensis, A. colossophilus and $A$. datchaensis have a paucispiral protoconch and are endemic to the Mediterranean Sea, and some of them have a restricted distribution. However, contrary to the above, some Alvania species (A. carina- ta, A. lactea) with paucispiral protoconch are widely distributed in the Atlantic and Arctic Oceans, and in the Mediterranean. Therefore, it may be concluded that the geographical distribution of a species cannot be explained on the basis of protoconch type only, which in fact may also depend on other ecological or historical features.

The shell dimension data obtained from the study were compared with those of Alvania species distributed along the Levantine coast of Turkey (Bitlis Bakır and Öztürk 2016), and some species (A. aspera, A. bozcaadensis, A. cimex, A. lactea and A. testae) from the Turkish Levantine coast were found to be taller than those of our study. However, the shells of A. discors, A. mamillata and A. scabra from the Levantine Sea were smaller than those originating from the Turkish Aegean coast.

According to the checklist published by Öztürk et al. (2014), 27 Alvania species were reported along the Turkish coasts, of which 24 are known to be distributed along the Aegean coast of Turkey; six of these species [A. campanii Tisselli and Giunchi, 2013, A. dorbignyi (Audouin, 1826), A. lanciae (Calcara, 1845), A. rudis (Philippi, 1844), A. settepassii Amati and Nofroni, 1985 and A. zetlandica (Montagu, 1816)] reported from the Turkish Aegean coast in earlier studies were not encountered in this study. To date, A. lanciae, $A$. rudis and $A$. zetlandica have only been reported from the Aegean coast of Turkey by Demir (2003). Yokeş and Demir (2013) reported A. settepassii along the Turkish coasts for the first time (Gökova Bay), but this species has not been recorded again from the Turkish coasts.

Although with some doubts, A. dorbignyi is the only Alvania species considered as alien. Its distribution in the Mediterranean Sea is limited to the eastern basin only: Cyprus (Bogi et al. 1989), Israel (Barash and Danin 1992, Delongueville and Scaillet 2007) and Tunisia (Campani 2009). The origin of A. dorbignyi is still unclear. Nordsieck (1972) suggested that this species originates from the Red Sea, but Mienis (1985) did not share this opinion, arguing that the species may have Indo-Pacific origin or is an anti-Lessepsian migrant. We found no specimens of the species, but it is clear that its distribution and origin need to be investigated in detail.

As a result of this study, the number of Alvania species distributed along the Aegean coast of Turkey has increased to 26 species with the addition of $A$. hispidula and A. marmarisensis n. sp. The Aegean Sea has fairly high biological diversity, due to its hydrographic characters and geomorphologic structures. Regarding the molluscan fauna, the Aegean coast of Turkey is the richest coast of Turkey (Öztürk et al. 2014).

\section{ACKNOWLEDGEMENTS}

The authors are much indebted to Bruno Amati (Roma, Italy) and Morena Giunchi (San Zaccaria (RA), Italy) for their advice and confirmation of some Alvania species, and to the editor and two anonymous referees for their constructive comments. 


\section{REFERENCES}

Albano P.G., Sabelli B. 2012. The molluscan assemblages inhabiting the leaves and rhizomes of a deep water Posidonia oceanica settlement in the central Tyrrhenian Sea. Sci. Mar. 76: 721-732.

Amati B. 2012. Alvania consociella Monterosato, 1884 junior synonym of Alvania lanciae (Calcara, 1845) (Prosobranchia, Rissoidae). Boll. Malacol. 48: 116-121.

Amati B. 2014. Description of Alvania alicea spec. nov. (Gastropoda, Rissoidae) from the Mediterranean Sea. Iberus 32: 87-95.

Amati B., Oliverio M. 1985. Alvania (Alvaniella hallgassi sp. n.) (Gastropoda; Prosobranchia). Notiz. CISMA 6: 28-34.

Amati B., Oliverio M. 1987. Alvania datchaensis sp. n. (Gastropoda; Prosobranchia). Notiz. CISMA 10: 46-53.

Antoniadou C., Koutsoubas D., Chintiroglou C. 2005. Mollusca fauna from infralittoral hard substrate assemblages in the North Aegean Sea. Belgian J. Zool. 135: 119-126.

Aslan-Cihangir H., Ovalis P. 2013. Seasonal variations and Structure of the Molluscan Assemblage in the Canakkale Strait (Turkey). Acta Zool. Bulgar 65: 233-250.

Avila S.P., Goud J., de Frias Martins A.M. 2012. Patterns of Diversity of the Rissoidae (Mollusca: Gastropoda) in the Atlantic and the Mediterranean Region. The Scientific World Journal 2012: Art. Id. $164890,30 \mathrm{pp}$ https://doi.org/10.1100/2012/164890

Bacescu M.C., Müller G.I., Gomoiu M.T. 1971. Ecologie Marina. Cercetari de Ecologie Bentala in Marea Neagra. Editura Academiei Republicii Socialiste Romania 4, 357 pp.

Barash A., Danin Z. 1992. Annotated List of Mediterranean Molluscs of Israel and Sinai. The Israel Academy of Sciences and Humanities, Jerusalem, $405 \mathrm{pp}$

Bitlis Bakır B., Öztürk B. 2016. Rissooidea Species distributed Along the Turkish Levantine Coast. Turk. J. Fish. Aquat. Sc. 16: 443-454.

Bitlis Bakır B., Öztürk B., Doğan A. et al. 2012. Mollusc Fauna of Iskenderun Bay with a Checklist of the Region. Turk. J. Fish. Aquat. Sci. 12: 171-184.

Bogi C., Cianfanelli S., Talenti E. 1989. Contributo alia conoscenza della malacofauna dell'isola di Cipro. In Nofroni L. (eds), Atti J Giornata di Studi Malacologici. C.I.S.M.A. Roma (1988), pp. 187-214.

Bouchet P., Warén A. 1993. Revision of the Northeast Atlantic bathyal and abyssal Mesogastropoda. Boll. Malacol. 3: 579-840.

Buzzurro G. 2003. A new species of Alvania from Cyprus (Gastropoda, Prosobranchia, Rissoidae). La Conchiglia 308: 43-46.

Buzzurro G., Greppi E. 1996. The Lessepsian molluscs of Taşucu (South-East Turkey). La Conchiglia 279: 3-22.

Buzzurro G., Prkic J. 2007. A new species of Alvania (Gastropoda: Prosobranchia: Rissoidae) from Croatian coast of Dalmatia. Triton 15: 5-9.

Campani E. 2009. An odd finding of Alvania dorbignyi (Gastropoda: Rissoidae). Mar. Biodiver. Rec. 2: 1-2 https://doi.org/10.1017/S1755267208000353

Cecalupo A., Quadri P. 1996. Contributo Alla Conoscenza Malacologica Per Il Nord Dell'Isola Di Cipro (part 3). Boll. Malacol. 31: $95-118$

Çınar M.E., Katağan T., Öztürk B., et al. 2012. Spatio temporal distributions of zoobenthos Mersin Bay (Levantine Sea, eastern Mediterranean) and the importance of alien species in benthic communities. Mar. Biol. Res. 8: 954-968. https://doi.org/10.1080/17451000.2012.706305

CLEMAM (Check List of European Marine Mollusca). 2016. Accessed 10.10.2016 at www.somali.asso.fr/clemam/index.php

Coan E. 1964. A Proposed Revision of the Rissoacean Families Rissoidae, Rissoinidae, and Cingulopsidae (Mollusca: Gastropoda). The Veliger 6: 164-171.

Criscione F., Ponder W.F., Köhler F., et al. 2016. A molecular phylogeny of Rissoidae (Caenogastropoda: Rissooidea) allows testing the diagnostic utility of morphological traits. Zool. J. Linn. Soc. London 179: 23-40. https://doi.org/10.1111/zoj.12447

Delongueville C., Scaillet R. 2007. Les espéces invasives de mollusques en Mediterranée. Novapex 8: 62

Demir M. 2003. Shells of Mollusca Collected from the Seas of Turkey. Turk. J. Zool 27: 101-140

Forbes E. 1844. Report on the Mollusca and Radiata of the Aegean Sea and on their distribution considered as bearing on Geology. In: Report of the 13th meeting of the British Association Advancement of Sciences, pp. 5-13.
Fretter V., Graham A. 1978. The Prosobranch Molluscs of Britain and Denmark Part. 4 Marine Rissoacea. J. Moll. Stud. 6: 54-241.

Gianuzzi-Savelli R., Pusateri F., Palmeri A., et al. 1997. Atlas of the Mediterranean Seashells (Caenogastropoda part 1: DiscopodaHeteropoda). Edizioni Evolver, Roma, 258 pp.

Gofas S. 1990. The littoral Rissoidae and Anabathridae of Sao Miguel, Azores. Acoreana 1990: 97-134.

Gofas S. 2007. Rissoidae (Mollusca: Gastropoda) from northeast Atlantic seamounts. J. Nat. Hist. 41: 779-885. https://doi.org/10.1080/00222930701298085

Gofas S., Moreno D., Salas C. 2011. Moluscos marinos de Andalucía: I. Introducción general, clase Solenogastres, clase Caudofoveata, clase Polyplacophora y clase Gastropoda (Prosobranchia). Servicio de Publicaciones e Intercambio Científico, Universidad de Málaga, Málaga. 342 pp.

Hoenselaar H.J., Goud J. 1998. The Rissoidae of the CANCAP expeditions, I: the genus Alvania Risso, 1826 (Gastropoda Prosobranchia). Basteria 62: 69-115.

Hudenbick B., Warén A. 1969. Smasnackor Vid Svenska Vastkusten 1. Slaktet Alvania. Sartryck ur Göteborgs Naturhistoriska Museums Arstryck 55-61.

Kocatas A. 1978. İzmir Körfezi Kayalık Sahillerinin Bentik Formları üzerinde Kalitatif ve Kantitatif Araştırmalar. Ege Üniversitesi Fen Fakültesi Monografiler Serisi. 12: 1-93.

Kocataș A., Bilecik N. 1992. Aegean Sea and its living resources. Water Products Research Institute, Bodrum. 88 pp.

Mienis H.K. 1985. Is Alvania dorbignyi (Audouin, 1826) A Lessepsian Migrant? Levantina 59: 652-654.

Nevill G. 1885. Hand list of Mollusca in the Indian Museum, Calcutta. Part II. Gastropoda, Prosobranchia-Neurobranchia (contd.). Government Printer, Calcutta. 306 pp.

Nordsieck F. 1972. Die europaischen Meeresschnecken (Opisthobranchia mit Pyramidellidae; Rissoacea). Gustav Fischer Verlag, Stuttgart, $327 \mathrm{pp}$.

Oberling J.J. 1960-1962. Une collection de microgastéropodes marins d'Attique. De l'annuaire du musée d'histoire naturelle, Part A. 207-221.

Oberling J.J. 1969-1971. On the littoral mollusca of the sea of Marmara. Jahrbuch des Naturhistorischen Museum, Bern, 4: 183-218.

Okuş E., Yükssek A., Yokeş M.B., et al. 2006. Coastal and Marine Biodiversity Assessment of Gökova Specially Protected Area Final Report. Environmental Protection Agency for Special Areas, Ministry of Environment and Forestry of Turkey.

Oliver J.D., Calvo M., Guallart J., et al. 2015. Gasterópodos marinos de las islas Chafarinas (Mediterráneo suroccidental). Iberus 33: $97-150$.

Oliverio M. 1986. Alvania amatii n. sp. (Gastropoda: Prosobranchia). Notiz. CISMA 7-8: 29-34.

Oliverio M. 1988. A new Prosobranch from the Mediterranean Sea, Alvania dianiensis n. sp. (Mollusca; Gastropoda). Bull. Zool. Mus. Univ. Amsterdam 11: 117-120.

Oliverio M., Amati B., Nofroni I. 1986. Proposta di Adeguamento Sistematico Dei Rissoidaea (sensu Ponder) Del Mar Mediterraneo Parte I: famiglia Rissoidae Gray, 1847 (Gastropoda: Prosobranchia). Notiz. CISMA 7-8: 35-52.

Ostroumoff A. 1894. K Estestvennoi istorii Bosfora. Prilojenie k LXXIV-my Tomu Zapisok Imper. Akademii Nauk, 5 pp.

Ostroumoff A. 1896. Otchet o dragirovkah i planktonniyh ulovaht ekspeditsia "Selyanika". Bull. Academie Imperiale des Sciences de St. Petersbourg 5: 33-92.

Öztürk B. 1998. Black Sea biological Diversity, Turkey. Black Sea Environmental Series No 9. United Nations Publish. 144 pp.

Öztürk B., Poutiers J.M., Önen M., et al. 2006. On the Occurrence of Rhomboidella prideaux (Leach, 1815) (Mollusca: Bivalvia: Mytilidae) in the Eastern Mediterranean. The Veliger 48: 215-219.

Öztürk B., Önen M., Doğan A. 2008. Türkiye Denizel Mollusca Türleri Tayin Atlas1, Ankara, 103T154 nolu Tübitak Projesi, 468 pp.

Öztürk B., Doğan A., Bitlis-Bakır B., et al. 2014. Marine Molluscs of the Turkish Coasts: An Updated Checklist. Turk. J. Zool. 38: $1-48$. https://doi.org/10.3906/zoo-1405-78

Pallary P.P. 1900. Coquilles Marines Du Littoral Du Departement D'oran. J. Conchyl 212-422.

Pitacco V., Orlando-Bonaca M., Mavric B. et al. 2014. Mollusc fauna associated with the Cystoseira algal associations in the Gulf of Trieste (Northern Adriatic Sea). Medit. Mar. Sci 15: 225-238. 
https://doi.org/10.12681/mms.466

Ponder W.E. 1985. A review of the genera of the Rissoidae (Mollusca: Mesogastropoda: Rissoacea). Rec. Aust. Mus. 4: 1-221. https://doi.org/10.3853/j.0812-7387.4.1985.100

Poursanidis D., Koutsoubas D. 2015. A computerized database (CorMol) on the molluscan fauna from the Mediterranean reef ecosystems: Part I, the coralligenous formations. Quat. Int. 390: 29-43. https://doi.org/10.1016/j.quaint.2015.07.029

Poursanidis D., Koutsoubas D., Arvanitidis C., et al. 2016. ReefMedMol: Mollusca from the infralittoral rocky shores - the biocoenosis of photophilic algae - in the Mediterranean Sea. Biodivers. Data J. 4: e7516. https://doi.org/10.3897/BDJ.4.e7516

Risso A. 1826. Histoire naturelle des principales productions de l'Europe Méridionale et particulièrement de celles des environs de Nice et des Alpes Maritimes. Vol. 4. Paris, Levrault, 439 pp.

Romani L. 2014. Alvania dalmatica Buzzurro et Prkic, 2007 (Gastropoda Rissoidae): range extension, shell variability, habitat and relationships with A. hallgassi Amati et Oliverio, 1985. Biodiver. J. 5: 509-514.

Scaperrotta M., Bartolini S., Bogi C. 2011. Accrescimenti: Stadi di accrescimento dei Molluschi marini del Mediterraneo. Vol. 3, $184 \mathrm{pp}$.

Tisselli M., Giunchi L. 2013. Due nuove specie di Alvania (Gastropoda: Rissoidae) dal nord-ovest della Turchia. Quad. Studi Nat. Romagna 37: 163-174.

Tringali L.P. 2001. Marine malacological records (Gastropoda, Prosobranchia, Heterobranchia, Opisthobranchia and Pulmonata) from Torres de Alcala, Mediterranean Marocco, with the description of a new philinid species. Boll. Malacol. 37: 207-222.

van Aartsen J.J.. 1982a. Synoptic tables of Mediterranean and European Conchology (Gen. Alvania). La Conchiglia 14: 20-21. van Aartsen J.J. 1982b. Synoptic tables of Mediterranean and European Conchology Genus Alvania: (Subg. Alvinia and Galeodina). La Conchiglia 14: 8-9.

van Aartsen J.J. 1982c. Sinoptic tables of Mediterranean and European Conchology Gen. Alvania: (Sbg.: Arsenia and Alvaniella). La Conchiglia 14: 4-6.

van Aartsen J.J., Kinzelbach R. 1990. Marine Molluscs from the İtuzu Beach near dalyan (Mediterranean coast of Turkey). Zool. Middle East 4: 103-112. https://doi.org/10.1080/09397140.1990.10637593

van Aartsen J.J., Menkhorst H.P.M.G., Gittenberger E. 1984. The marine Mollusca of the Bay of Algeciras, Spain, with general notes on Mitrella, Marginellidae and Turridae. Basteria Supplement 2: 1-135.

van Aartsen J.J., Barash A., Carrozza F. 1989. Addition to the Knowledge of the Mediterranean Mollusca of Israel and Sinai. Boll. Malacol. 25: 63-76.

Verduin A. 1984. On the taxonomy of some Recent European marine species of the genus Cingula s.l. (Gastropoda: Prosobranchia). Basteria 48: 37-87.

Verduin A. 1986. Alvania cimex (L.) s.l. (Gastropoda, Prosobranchia), an aggregate species. Basteria 50: 25-32.

Warén A. 1974. Revision of the Arctic-Atlantic Rissoidae (Gastropoda, Prosobranchia). Zool. Scripta 3: 121-135. https://doi.org/10.1111/j.1463-6409.1974.tb00810.x

World Register of Marine Species. 2016. WORMS web page. (accessed 10.10.2016) WWW.marinespecies.org

Yokes M.B., Demir V. 2013. Ayvalık Adaları Tabiat Parkı Denizel Biyolojik Çeşitlilik Çalışması. Teknik rapor Serisi 20: 104.

Zenetos A., van Aartsen J.J. 1995. The deep sea Molluscan Fauna of the S.E. Aegean Sea and its relation to the neighbouring faunas. Boll. Malacol. 30: 253-268. 